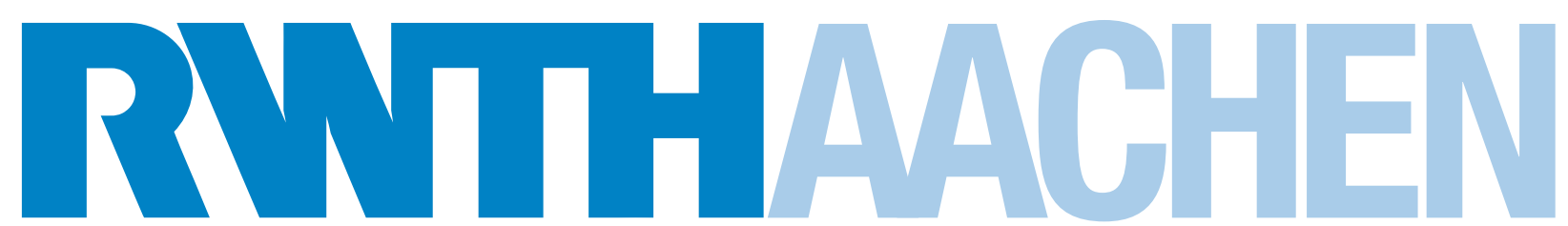

RHEINISCH-WESTFÄLISCHE TECHNISCHE HOCHSCHULE AACHEN

\title{
Institut für Mathematik
}

On minimal immersions in Finsler space.

\author{
by \\ P. Overath \\ H. von der Mosel
}

Report No. 72

April

2014

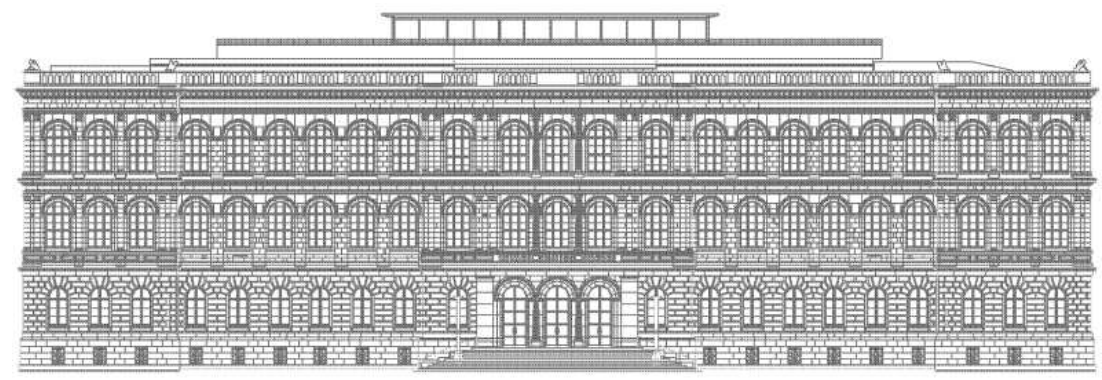

Institute for Mathematics, RWTH Aachen University

Templergraben 55, D-52062 Aachen

Germany 


\title{
On minimal immersions in Finsler space.
}

\author{
Patrick Overath, Heiko von der Mosel
}

April 1, 2014

\begin{abstract}
We explore a connection between the Finslerian area functional and well-investigated Cartan functionals to prove new Bernstein theorems, uniqueness and removability results for Finslerminimal graphs, as well as enclosure theorems and isoperimetric inequalities for minimal immersions in Finsler spaces. In addition, we establish the existence of smooth Finsler-minimal immersions spanning given extreme or graphlike boundary contours.
\end{abstract}

Mathematics Subject Classification (2000): 44A12, 49Q05, 49Q10, 53A35, 53B40, 53C60

\section{Introduction}

\subsection{Minimal immersions in Finsler geometry}

Minimal surface theory in Finsler spaces seems to be a largely underdeveloped terrain. Recently, we established a new connection between Finsler-minimal immersions and anisotropic variational integrals, so-called Cartan functionals, to treat the Plateau problem in Finsler 3-space; see [28]. In the present note we explore this connection to substantially extend the few known results about minimal graphs, available only in very specific Finsler-Minkowski spaces so far, and we prove new global results for Finsler-minimal immersions such as enclosure theorems and isoperimetric inequalities. In addition, we establish the existence of smooth Finsler-minimal immersions spanning given extreme or graphlike boundary contours. Our general assumption on the Finsler structure turns out to be natural and sharp, since it translates to known sharp threshold values for the anisotropies of the few specific Finsler-Minkowski spaces investigated so far.

In order to briefly recall the precise notion of Finsler-minimal immersions (in the sense of Busemann and Hausdorff) let $\mathscr{N}=\mathscr{N}^{n}$ be an $n$-dimensional smooth manifold with tangent bundle $T \mathscr{N}:=\bigcup_{x \in \mathscr{N}} T_{x} \mathscr{N}$ and its zero-section $o:=\{(x, 0) \in T \mathscr{N}\}$. A non-negative function $F \in$ $C^{\infty}(T \mathscr{N} \backslash o)$ is called a Finsler metric on $\mathscr{N}$ (so that $(\mathscr{N}, F)$ becomes a Finsler manifold) if $F$ satisfies the conditions

(F1) $F(x, t y)=t F(x, y)$ for all $t>0$ and all $(x, y) \in T \mathscr{N}$ (homogeneity);

(F2) $g_{i j}(x, y):=\left(F^{2} / 2\right)_{y^{i} y^{j}}(x, y)$ form the coefficients of a positive definite matrix, the fundamental tensor, for all $(x, y) \in T \mathscr{N} \backslash o$, where for given local coordinates $x^{1}, \ldots, x^{n}$ about $x \in \mathscr{N}$, the $y^{i}, i=1, \ldots, n$, denote the corresponding bundle coordinates via $y=\left.y^{i} \frac{\partial}{\partial x^{i}}\right|_{x} \in T_{x} \mathscr{N}$. Here we sum over repeated Latin indices from 1 to $n$ according to the Einstein summation convention, and $F(x, y)$ is written as $F\left(x^{1}, \ldots, x^{n}, y^{1}, \ldots, y^{n}\right)$. 
If $F(x, y)=F(x,-y)$ for all $(x, y) \in T \mathscr{N}$ then $F$ is called a reversible Finsler metric, and if $F$ on $\mathscr{N}=\mathbb{R}^{n}$ depends only on $y$ in standard coordinates, then $F$ is called a Minkowski metric. Any $C^{2}-$ immersion $X: \mathscr{M}^{m} \hookrightarrow \mathscr{N}^{n}$ from a smooth $m$-dimensional manifold $\mathscr{M}=\mathscr{M}^{m}$ into $\mathscr{N}$ induces a pulled-back Finsler metric $X^{*} F$ on $\mathscr{M}$ via

$$
\left(X^{*} F\right)(u, v):=F\left(X(u),\left.d X\right|_{u}(v)\right) \quad \text { for }(u, v) \in T \mathscr{M} .
$$

Following Busemann [5] and Shen [32] we define the Busemann-Hausdorff volume form as the volume ratio of the Euclidean and the Finslerian unit ball, i.e.,

$$
d \operatorname{vol}_{X^{*} F}(u):=\sigma_{X^{*} F}(u) d u^{1} \wedge \ldots \wedge d u^{m} \quad \text { on } \mathscr{M},
$$

where

$$
\sigma_{X^{*} F}(u):=\frac{\mathscr{H}^{m}\left(B_{1}^{m}(0)\right)}{\mathscr{H}^{m}\left(\left\{v=\left(v^{1}, \ldots, v^{m}\right) \in \mathbb{R}^{m}: X^{*} F\left(u, v^{\delta} \frac{\partial}{\left.\partial u^{\delta}\right|_{u}}\right) \leq 1\right\}\right.},
$$

with a summation over Greek indices from 1 to $m$ in the denominator. Here $\mathscr{H}^{m}$ denotes the $m$ dimensional Hausdorff-measure. The Busemann-Hausdorff area or in short Finsler area ${ }^{1}$ of the immersion $X: \mathscr{M} \rightarrow \mathscr{N}$ is then given by

$$
\operatorname{area}_{\Omega}^{F}(X):=\int_{u \in \Omega} d \operatorname{vol}_{X^{*} F}(u)
$$

for a measurable subset $\Omega \subset \mathscr{M}$. Shen [32, Theorem 1.2] derived the first variation of this functional which leads to the definition of Finsler-mean curvature. Critical immersions for area $F_{\Omega}^{F}$ are therefore Finsler-minimal immersions, or simply minimal hypersurfaces in $(\mathscr{N}, F)$ if the co-dimension $n-m$ equals 1.

By means of his variational formulas Shen excluded the existence of closed oriented Finslerminimal submanifolds in Minkowski space, i.e., in $\mathbb{R}^{n}$ equipped with a Minkowski metric $F=F(y)$; see [32, Theorem 1.3]. Souza and Tenenblatt proved in [36] that there is up to homotheties exactly one complete embedded Finsler-minimal surface of revolution in the Minkowski-Randers space $\left(\mathbb{R}^{3}, F\right)$ where $F(y):=|y|+b_{i} y^{i}$ for some constant vector $b$ with length $|b|<1$. In the same setting Souza, Spruck, and Tenenblatt [35] computed the fairly complicated pde for Finsler-minimal graphs, and proved under the more restrictive bound $|b|<1 / \sqrt{3}$ that any Finsler-minimal graph defined on the entire plane $\mathbb{R}^{2}$ is an affine plane. This bound on $|b|$ is actually sharp for this Bernstein theorem: beyond the threshold value, i.e., for $|b| \in(1 / \sqrt{3}, 1)$, where $\left(\mathbb{R}^{3}, F\right)$ is still a Finsler space (see e.g. [7, p. 4]), the pde ceases to be elliptic, and there exists a Finsler-minimal cone with a point singularity in that case; see [36, Proposition 13] and [35, p. 300]. Souza et al. also proved a touching principle and the removability of isolated singularities of Finsler-minimal graphs in this specific three-dimensional Minkowski-Randers space. Their Bernstein theorem was later generalized by Cui and Shen [12] to entire $m$-dimensional Finsler-minimal graphs in the more general $(\alpha, \beta)$-Minkowski spaces $\left(\mathbb{R}^{m+1}, F\right)$. Here, $F(y)$ equals $\alpha(y) \phi[\beta(y) / \alpha(y)]$ with $\alpha(y):=|y|$ and the linear perturbation term $\beta(y):=b_{i} y^{i}$, where $\phi$ is a positive smooth scalar function satisfying a particular differential equation to guarantee that $F$ is at least a Finsler metric; see e.g. [7, Lemma 1.1.2]. Cui and Shen require for their Bernstein results fairly complicated additional and more restrictive conditions on $\phi$ (see condition (1) in [12, Theorem 1.1] or condition (4) of [12, Theorem 1.2]) that could be verified

\footnotetext{
${ }^{1}$ Notice that the alternative Holmes-Thompson volume form (see [1]) leads to a different notion of Finslerian minimal surfaces that we do not address here.
} 
only for a few specific choices of $(\alpha, \beta)$-metrics, and only in dimension $m=2$ : for the MinkowskiRanders case with $\phi(s)=1+s$ if $|b|<1 / \sqrt{3}$ (reproducing [35, Theorem 6]), for the two-order metric with $\phi(s)=(1+s)^{2}$ under the condition $|b|<1 / \sqrt{10}$, or for the Matsumoto metric where $\phi(s)=(1-s)^{-1}$ if $|b|<1 / 2$. Also these threshold values for $|b|$ are sharp; one finds Finsler-minimal cones for $|b|$ beyond the respective bounds for the Minkowski-Randers and for the two-order metric. And the Matsumoto metric simply ceases to be a Finsler metric if $|b|>1 / 2$.; see [12, Theorem 5.3 $\&$ Section 6]. Cui and Shen [13] derived a representation formula for rotationally symmetric Finslerminimal surfaces in Minkowski- $(\alpha, \beta)$-spaces and they presented a unique explicit forward-complete rotationally symmetric Finsler-minimal surface in Minkowski-Randers-3-space.

Our results presented in the next subsection generalize and extend many of these results to general Finsler-Minkowski spaces under a natural assumption on a suitable symmetrization of the underlying Finsler metric. It turns out that this assumption reproduces the sharp bounds on the anisotropy $|b|$ in the specific Finsler-Minkowski-spaces described above.

For any function $F \in C^{0}(T \mathscr{N} \backslash o)$ that is positively 1-homogeneous in the $y$-variable, we define the $m$-harmonic symmetrization $F_{\text {sym }}$ as

$$
F_{\text {sym }}(x, y):=\left[\frac{2}{\frac{1}{F^{m}(x, y)}+\frac{1}{F^{m}(x,-y)}}\right]^{\frac{1}{m}} \quad \text { for } \quad(x, y) \in T \mathscr{N} \backslash o,
$$

which by definition is even and positively 1-homogeneous in the $y$-variable, and thus continuously extendible by zero to the whole tangent bundle $T \mathscr{N}$. But even if $F$ is a Finsler metric, the symmetrized form $F_{\text {sym }}$ might not be, which motivates our General Assumption:

(GA) Let $F(x, y)$ be a Finsler metric on $\mathscr{N}=\mathbb{R}^{m+1}$ (with respect to its standard coordinates) such that its $m$-harmonic symmetrization $F_{\mathrm{sym}}(x, y)$ is also a Finsler metric on $\mathbb{R}^{m+1}$.

Notice that a reversible Finsler metric $F$ automatically coincides with its $m$-harmonic symmetrization $F_{\text {sym }}$ so that our general assumption (GA) is superfluous in reversible Finsler spaces. A sufficient criterion guaranteeing that (GA) holds for non-reversible Finsler metrics was derived in [28, Theorem 1.5], allowing for a non-trivial $x$-dependence such as $F(x, y):=F_{\text {rev }}(x, y)+b_{i} y^{i}$ where $F_{\text {rev }}$ is a reversible Finsler metric. Even in the Minkowski setting this creates examples that were not treated before, for example, choosing the perturbed quartic metric (see [3, p. 15])

$$
F_{\mathrm{rev}}(y):=\sqrt{\sqrt{\sum_{i=1}^{m+1}\left(y^{i}\right)^{4}}+\epsilon \sum_{i=1}^{m+1}\left(y^{i}\right)^{2}} \quad \text { for } \epsilon>0
$$

as the reversible part of $F(y)=F_{\text {rev }}(y)+b_{i} y^{i}$.

\subsection{Main results}

Finsler-minimal graphs. As Finsler-minimal graphs we denote $m$-dimensional Finsler-minimal immersions that can be written as a graph over some domain in a hyperplane of the ambient space $\mathbb{R}^{m+1}$. Such a graph is called entire if the domain is the whole hyperplane.

Theorem 1.1 (Bernstein theorems). Let $F=F(y)$ be a Minkowski metric on $\mathbb{R}^{m+1}$ satisfying assumption (GA). Then the following holds:

(i) If $m=2$ or $m=3$, then every entire Finsler-minimal graph is an affine plane. 
(ii) For every $m \leq 7$ there exists a constant $\delta=\delta(m)>0$ such that every entire Finsler-minimal graph is an affine plane if

$$
\min \left\{\|F(\cdot)-|\cdot|\|_{C^{3}\left(\mathbb{S}^{m}\right)},\left\|F_{\text {sym }}(\cdot)-|\cdot|\right\|_{C^{3}\left(\mathbb{S}^{m}\right)}\right\} \leq \delta .
$$

(iii) For every $m \in \mathbb{N}$ and $\gamma \in(0,1)$ there is a constant $\delta=\delta(m, \gamma)>0$ such that every entire Finsler-minimal graph $\left.\{u, f(u)): u \in \mathbb{R}^{m}\right\} \subset \mathbb{R}^{m+1}$ is an affine plane if

$$
\min \left\{\|F(\cdot)-|\cdot|\|_{C^{3}\left(\mathbb{S}^{m}\right)},\left\|F_{\text {sym }}(\cdot)-|\cdot|\right\|_{C^{3}\left(\mathbb{S}^{m}\right)}\right\} \leq \delta,
$$

and if it satisfies the additional growth condition

$$
|D f(u)|=O\left(\mid\left(u,\left.f(u)\right|^{\gamma}\right) \quad \text { as }|u| \rightarrow \infty\right.
$$

on the gradient $D f(u)=\left(f_{u^{1}}, \ldots, f_{u^{m}}\right)$, where $u=\left(u^{1}, \ldots, u^{m}\right) \in \mathbb{R}^{m}$.

Part (i) is apparently new for $m=3$, and it generalizes [35, Theorem 6] and [12, Theorem 1.2] for $m=2$ to general Finsler-Minkowski spaces. Indeed, calculating what (GA) implies for the specific Finsler metrics investigated in [35] and [12, Section 6], we obtain exactly the threshold values for the anisotropy $|b|$ mentioned above. Part (ii) generalizes the part of [12, Theorem 1.1] dealing with Finsler-minimal graphs with respect to the Busemann-Hausdorff-area, since the given scalar function $\phi(s):=(1+h(s))^{-1 / m}$ with an arbitrary odd smooth function $h: \mathbb{R} \rightarrow(-1,1)$ leads via an easy computation to an $(\alpha, \beta)$-Minkowski metric satisfying (GA). Part (iii) for arbitrary dimensions $m$ is, to the best of our knowledge, completely new in the context of Finsler-minimal immersions.

The next two results deal with uniqueness of Finsler-minimal graphs and the removability of singularities.

Theorem 1.2 (Uniqueness). Let $F=F(y)$ be a Minkowski metric on $\mathbb{R}^{m+1}$ satisfying assumption (GA), and assume that $f_{1}, f_{2} \in C^{0}(\bar{\Omega} \backslash K) \cap C^{2}(\Omega \backslash K)$ are functions that define two Finsler-minimal graphs over $\Omega \backslash K \subset \mathbb{R}^{m}$, where $\Omega$ is a bounded domain with a $C^{1}$-boundary $\partial \Omega$, and $K \subset \Omega$ is compact with $\mathscr{H}^{m-1}(K)=0$ such that $\Omega \backslash K$ is connected. Then, equality of $f_{1}$ and $f_{2}$ on the boundary $\partial \Omega$ implies $f_{1}=f_{2}$ on $\bar{\Omega} \backslash K$.

The only known uniqueness result for Finsler-minimal graphs we are aware of is contained in the work of Souza et al. [35, Corollary 7] for the specific choice of a Randers-Minkowski 3-space, which can be recovered from Theorem 1.2 by choosing $m=2$ and $K=\emptyset$.

Theorem 1.3 (Removability of singularities). Let $F=F(y)$ be a Minkowski metric on $\mathbb{R}^{m+1}$ satisfying assumption (GA), and assume that $\Omega \subset \mathbb{R}^{m}$ is an arbitrary domain and $K$ is a locally compact subset of $\Omega$ with $\mathscr{H}^{m-1}(K)=0$. Then any Finsler-minimal graph of class $C^{2}$ on $\Omega \backslash K$ can be extended as a Finsler-minimal graph of class $C^{2}$ onto all of $\Omega$.

With this result we generalize the only known removability result for Finsler-minimal graphs [35, Proposition 12], which can be reproduced from Theorem 1.3 by setting $m=2, K:=\left\{u_{0}\right\}$ for some $u_{0} \in \Omega$, and choosing the particular Minkowski-Randers metric $F(y):=|y|+b_{i} y^{i}$ with $|b|<1 / \sqrt{3}$.

Recall that our general assumption (GA) in Theorems 1.1 and 1.3 is sharp since it translates to the sharp bounds on $|b|$ established in the work of Souza et al. and Cui and Shen in the specific Randers and $(\alpha, \beta)$-spaces. The minimal cones constructed in the respective spaces for $|b|$ above the threshold values demonstrate their sharpness; see [35, p. 300] and [12, Theorem 5.3]. 
Global results for Finsler-minimal immersions. Apart from Shen's nonexistence result for closed oriented Finsler-minimal submanifolds in Minkowski space [32, Theorem 1.3] and a few global results on rotationally symmetric Finsler-minimal surfaces [36], [13] in specific $(\alpha, \beta)$-spaces, there seems to be not much known about the global behaviour of Finsler-minimal immersions that are not graphs. We present here a simple enclosure theorem - well known for classic minimal surfaces in Euclidean space - and a selection of isoperimetric inequalities for Finsler-minimal immersions $X: \mathscr{M} \rightarrow \mathscr{N}:=$ $\mathbb{R}^{m+1}$, where $\mathscr{M}$ denotes a smooth oriented $m$-dimensional manifold with boundary $\partial \mathscr{M}$.

Theorem 1.4 (Convex hull property). Let $F=F(y)$ be a Minkowski metric on $\mathbb{R}^{m+1}$ such that assumption $(\mathrm{GA})$ holds. Then the image $X(\mathscr{M})$ of any Finsler-minimal immersion

$$
X \in C^{2}\left(\operatorname{int}(\mathscr{M}), \mathbb{R}^{m+1}\right) \cap C^{0}\left(\mathscr{M}, \mathbb{R}^{m+1}\right)
$$

is contained in the convex hull of its boundary $X(\partial \mathscr{M})$, where $\operatorname{int}(\mathscr{M})=\mathscr{M} \backslash \partial \mathscr{M}$.

For a general Finsler metric $F=F(x, y)$ we set

$$
M_{F}:=\sup _{\mathbb{R}^{m+1} \times \mathbb{S}^{m}} F(\cdot, \cdot) \text { and } m_{F}:=\inf _{\mathbb{R}^{m+1} \times \mathbb{S}^{m}} F(\cdot, \cdot) .
$$

Notice that for a Minkowski metric one automatically has $0<m_{F} \leq M_{F}<\infty$, since (F1) and (F2) imply that $F>0$. A simple variant of the isoperimetric inequality of the form

$$
\operatorname{area}^{F}(X) \leq \frac{M_{F}^{2}}{4 \pi m_{F}^{2}}\left(\mathscr{L}^{F}(\Gamma)\right)^{2}
$$

has been shown for (possibly branched) Finsler-area minimizing surfaces in $\mathbb{R}^{3}$ with a given boundary contour $\Gamma \subset \mathbb{R}^{3}$ by a simple comparison with classic minimal surfaces; see [28, Corollary 1.3]. Here, $\mathscr{L}^{F}(\Gamma):=\int F(\Gamma, \dot{\Gamma})$ denotes the Finslerian length of $\Gamma$.

For the following isoperimetric inequalities for Finsler-minimal immersions in Finsler space denote by $d S_{F}$ the volume form on the boundary $\partial \mathscr{M}$ induced by $X$ whose restriction $\left.X\right|_{\partial \mathscr{M}}: \partial \mathscr{M} \rightarrow$ $\mathbb{R}^{m+1}$ is again an immersion. Analogous to (1.1) one defines

$$
\int_{\omega} d S_{F}:=\operatorname{area}_{\omega}^{F}\left(\left.X\right|_{\partial \mathscr{M}}\right)
$$

for any relatively open set $\omega \subset \partial \mathscr{M}$. For the special choice $F(\cdot)=|\cdot|$, we set $\int_{\Omega} d S:=\int_{\Omega} d S_{F}$.

Theorem 1.5 (Isoperimetric inequalities). Let $F=F(x, y)$ be a Finsler metric on $\mathbb{R}^{m+1}$ satisfying assumption $(\mathrm{GA})$ and let $X \in C^{2}\left(\operatorname{int}(\mathscr{M}), \mathbb{R}^{m+1}\right) \cap C^{1}\left(\mathscr{M}, \mathbb{R}^{m+1}\right)$ be a Finsler-minimal immersion. Then the following holds.

(i) If $F=F(y)$ and $X(\partial \mathscr{M}) \subset \overline{B_{R}^{F}(a)}$ for some $a \in \mathbb{R}^{m+1}$, where $\overline{B_{R}^{F(a)}}$ denotes the closed Finsler-Minkowski unit ball $\left\{v \in \mathbb{R}^{m+1}: F(v-a) \leq R\right\}$, then

$$
\operatorname{area}_{\mathscr{M}}^{F}(X) \leq \frac{R}{m}\left(\frac{M_{F}}{m_{F}}\right)^{m} \sqrt{1+\Lambda(F)(m+1)\left(m / m_{F}\right)^{2}} \int_{\partial \mathscr{M}} d S_{F},
$$

where $\Lambda(F)$ denotes the largest possible eigenvalue of the fundamental tensor $\left(g_{i j}\right)$ of $F$ when restricted to the sphere $\mathbb{S}^{m}$. 
(ii) If $m=2$ and $F=F(y)$, and if the boundary $\partial \mathscr{M}$ consists of $k \geq 1$ closed rectifiable Jordan curves $\gamma_{i}$ with images $\Gamma_{i}:=X\left(\gamma_{i}\right)$ for $i=1, \ldots, k$, then for all $a \in \mathbb{R}^{3}$

$$
\operatorname{area}_{\mathscr{M}}^{F}(X) \leq \frac{M_{F}^{2}}{m_{F}^{2}} \sqrt{1+\Lambda(F) \frac{12}{m_{F}^{2}}} \sum_{i=1}^{k}\left[\frac{\mathscr{L}^{F}\left(\Gamma_{i}\right)^{2}}{4 \pi}+\frac{1}{2} \mathscr{L}^{F}\left(\Gamma_{i}\right) \operatorname{dist}_{F}\left(a, \Gamma_{i}\right)\right],
$$

where $\operatorname{dist}_{F}$ denotes the Finslerian distance in $\mathbb{R}^{3}$ induced by $F$, and where $\Lambda(F)$ is as in part (i).

(iii) Let $m=2, \mathscr{M}=B \equiv B_{1}(0) \subset \mathbb{R}^{2}, X \in C^{2, \alpha}\left(\bar{B}, \mathbb{R}^{3}\right)$ with $\|X\|_{L^{\infty}\left(B, \mathbb{R}^{3}\right)} \leq 1$, mapping $\partial B$ topologically onto the closed Jordan curve $\Gamma \subset B_{R}(a) \subset \mathbb{R}^{3}$ for some a $\in \mathbb{R}^{3}, R \leq 1$. Then there is a universal constant $\delta>0$ such that

$$
\operatorname{area}_{B}^{F}(X) \leq R\left\{c_{1}(F) M_{F}^{* 2}\left[\int_{\Gamma} \kappa d s-2 \pi\right]+c_{2}(F) \frac{M_{F}^{* 2}}{m_{F}^{*}} \mathscr{L}^{F}(\Gamma)\right\},
$$

as long as

$$
\min \left\{\|F(\cdot)-|\cdot|\|_{C^{3}\left(\overline{B_{1}(0)} \times \mathbb{S}^{m}\right)},\left\|F_{\mathrm{sym}}(\cdot)-|\cdot|\right\|_{C^{3}\left(\overline{B_{1}(0)} \times \mathbb{S}^{m}\right)}\right\} \leq \delta
$$

holds. Here $M_{F}^{*}$ and $m_{F}^{*}$ denote the supremum and the infimum of $F$ on $\overline{B_{1}(0)} \times \mathbb{S}^{m}$, respectively, and $\int_{\Gamma} \kappa d s$ is the Euclidean total curvature of the boundary curve $\Gamma$.

Notice for parts (i) and (ii) that $M_{F}=m_{F}=\Lambda(F)=1$ if $F$ equals the Euclidean norm, that is, $F(x, y)=|y|$. Likewise in part (iii) one can show, that the constant $c_{1}(F)$ vanishes and $c_{2}(F)=1 / 2$, and $M_{F}^{*}=m_{F}^{*}=1$, if $F$ is the Euclidean norm. On the other hand, if the boundary contour $\Gamma$ happens to be planar and convex, then according to Fenchel's theorem the first summand vanishes in (1.9) and one is left with a fairly simple isoperimetric inequality as long as $F$ satisfies (1.10).

Existence and uniqueness of Finsler-minimal immersions spanning given boundary contours. For a Finsler metric $F=F(x, y)$ on $\mathbb{R}^{3}$ satisfying our general assumption (GA) and for a given rectifiable Jordan curve $\Gamma \subset \mathbb{R}^{3}$, we could establish in [28, Theorem 1.2] the existence of conformally parametrized minimizers of $\operatorname{area}_{B}^{F}$ in the class of Sobolev mappings $W^{1,2}\left(B, \mathbb{R}^{3}\right)$ from the two-dimensional unit ball $B=B_{1}(0) \subset \mathbb{R}^{2}$ into $\mathbb{R}^{3}$ that parametrize $\Gamma$ on $\partial B$ in a weakly monotonic way $^{2}$. The proof produces a mild improvement in regularity of the $\operatorname{area}_{B}^{F}$-minimizer, i.e., continuity up to the boundary, interior Hölder continuity and a slightly better integrability of the gradient, but in general there is up to now no way to exclude branch points, or even to estimate the size of the set of branch points. These are points, where the Jacobian of the mapping fails to have rank two. If one asks for immersed or even embedded Finsler-minimal surfaces spanning a given boundary contour one has to add more assumptions on the boundary curve.

Theorem 1.6 (Existence). Let $F=F(x, y)$ be a Finsler metric on $\mathbb{R}^{3}$ satisfying assumption (GA).

(i) If $\Gamma \subset \mathbb{R}^{3}$ is a smooth closed Jordan curve contained in the boundary of a strictly Finsler area mean convex body, then for each $g \geq 0$ there exists a smooth embedded Finsler-minimal surface spanning $\Gamma$ with genus less or equal $g$. In particular, such an embedded Finsler-minimal surface exists if $F=F(y)$ and if $\Gamma$ is contained in the boundary of a strictly convex body.

\footnotetext{
${ }^{2}$ See [14, pp. 231-232] for the notion of weakly monotonic mappings on the boundary.
} 
(ii) If $F=F(y)$ and $\Gamma$ is a graph of bounded slope over $\partial \Omega$ for some bounded convex domain $\Omega \subset \mathbb{R}^{2}$, then there is a smooth and (up to reparametrizations) unique Finsler-minimal graph spanning $\Gamma$.

Roughly speaking, a body is Finsler mean convex if inward variations of its boundary lead to an infinitesimal decrease of Finsler area; for details we refer to Section 3. The bounded slope condition in the last part of the theorem means that we find a constant $R>0$, so that we can write $\Gamma$ as a graph,

$$
\Gamma=\left\{(u, \gamma(u)) \in \mathbb{R}^{3}: u=\left(u^{1}, u^{2}\right) \in \partial \Omega\right\}
$$

for some function $\gamma: \partial \Omega \rightarrow \mathbb{R}$, such that for any curve point $\left(u_{0}, \gamma\left(u_{0}\right)\right) \in \Gamma$ there exist two vectors $p_{0}^{+}, p_{0}^{-} \in \overline{B_{R}(0)} \subset \mathbb{R}^{2}$ such that the two affine linear functions

$$
\ell_{0}^{+}(u):=p_{0}^{+} \cdot\left(u-u_{0}\right)+\gamma\left(u_{0}\right) \text { and } \ell_{0}^{-}(u):=p_{0}^{-} \cdot\left(u-u_{0}\right)+\gamma\left(u_{0}\right)
$$

satisfy $\ell_{0}^{-}(u) \leq \gamma(u) \leq \ell_{0}^{+}(u)$ for all $u \in \partial \Omega$. In particular, if $\Omega$ is strictly convex and $\Gamma$ is a $C^{2}$-graph over $\partial \Omega$ then $\Gamma$ satisfies the bounded slope condition; see [19, pp. 309, 310].

For part (i) of Theorem 1.6 one can even prescribe an upper bound $G \geq 0$ on the genus of the Finsler-minimal embedding, which itself is Finsler-area minimizing among all embedded surfaces with the same boundary and with genus less or equal $G$.

Strategy of proofs and structure of the paper. The key to proving all these results is a connection between Finsler minimal surfaces and so-called Cartan functionals, i.e., parameter invariant variational integrals with a specific structure of the integrand. This connection has been established in [28] by means of the spherical Radon transform. We will recall all relevant facts on Cartan functionals and on their relation to Finsler minimal immersions in the next section; see Section 2.1. In addition, in order to apply the existing theory on Cartan functionals we need to strengthen this connection to show that Finsler area generates an elliptic Cartan integrands, that is, a strict parametric convexity property that was not necessary for the weak existence theory for the Plateau problem presented in [28]. Here, however, we need to analyse the behaviour of the spherical Radon transform on a suitable function space endowed with a Fréchet topology; see Section 2.2, in particular Corollary 2.14 ensuring elliptic Cartan integrands related to Finsler area. In Section 3 we apply various results on critical immersions of Cartan functionals established by H. Jenkins [26], L. Simon [34], [33], B. White [37], U. Clarenz and the second author [9], [10], [11], S. Winklmann [38], [40], [39], and S. Hildebrandt and F. Sauvigny, [23] to prove the results stated above.

Acknowledgments. Substantial parts of this work are contained in the first author's thesis who was partially supported by DFG grant no. Mo 966/3-1,2, and moreover by the Excellence Initiative of the German federal and state governments. Moreover, the second author would like to express his gratitude to Professor Seiki Nishikawa for inviting him to Tohoku University at Sendai, Japan, to give a series of talks on this line of research.

\section{Finsler-minimal immersions from a variational viewpoint}

\subsection{Finsler area and Cartan functionals}

The explicit form (1.1) of the Busemann-Hausdorff volume implies that bounds on the Finsler metric directly transfer to corresponding bounds on the area functional. 
Lemma 2.1 (Area comparison). Let $F=F(x, y)$ be a Finsler metric on $\mathbb{R}^{n}$, and denote by $E=$ $E(y):=|y|$ the Euclidean metric on $\mathbb{R}^{n}$. Assume that there are two constants $0<m_{F} \leq M_{F}$ such that

$$
m_{F}|y|=m_{F} E(y) \leq F(x, y) \leq M_{F} E(y)=M_{F}|y| \quad \text { for all }(x, y) \in \mathbb{R}^{n} \times \mathbb{R}^{n} .
$$

Then one has

$$
m_{F}^{k} \operatorname{area}_{\Omega}^{E}(X) \leq \operatorname{area}_{\Omega}^{F}(X) \leq M_{F}^{k} \operatorname{area}_{\Omega}^{E}(X)
$$

for every immersion $X: \Sigma^{k} \rightarrow \mathbb{R}^{n}$ from a smooth $k$-dimensional manifold $\Sigma$ into $\mathbb{R}^{n}$ and for every open set $\Omega \subset \Sigma$.

Proof: Inequality (2.1) implies $m_{F} X^{*} E(v) \leq X^{*} F(u, v) \leq 1$ for all $u \in \Omega$ and all $v \in$ $\left\{v=\left(v^{1}, \ldots, v^{k}\right) \in T_{u} \Omega \simeq \mathbb{R}^{k}: X^{*} F(u, v) \leq 1\right\}$. Hence we get

$$
\left\{v \in \mathbb{R}^{k}: X^{*} F(u, v) \leq 1\right\} \subset\left\{v \in \mathbb{R}^{k}: X^{*} E(u, v) \leq \frac{1}{m_{F}}\right\} .
$$

The $k$-dimensional Hausdorff-measure is monotonic and scales like $\mathscr{H}^{k}(t A)=t^{k} \mathscr{H}^{k}(A)$ for all $t>0$ and for any measurable set $A \subset \mathbb{R}^{n}$; see, e.g., [15, Chapter 2.1, Theorem 2]. Therefore (2.3) yields

$$
\begin{aligned}
\mathscr{H}^{k}\left(\left\{v \in \mathbb{R}^{k}: X^{*} F(u, v) \leq 1\right\}\right) & \leq \mathscr{H}^{k}\left(\left\{v \in \mathbb{R}^{k}: X^{*} E(u, v) \leq \frac{1}{m_{F}}\right\}\right) \\
& =\mathscr{H}^{k}\left(\frac{1}{m_{F}}\left\{v \in \mathbb{R}^{k}: X^{*} E(u, v) \leq 1\right\}\right) \\
& =\frac{1}{m_{F}^{k}} \mathscr{H}^{k}\left(\left\{v \in \mathbb{R}^{k}: X^{*} E(u, v) \leq 1\right\}\right) .
\end{aligned}
$$

Combining (2.4) with (1.1) leads to $m_{F}^{k} \sigma_{X^{*} E}(u) \leq \sigma_{X^{*} F}(u)$, and by means of the definition (1.2) of Finsler area we thus obtain the left inequality in (2.2). The second estimate in (2.2) can be shown analogously by exchanging the role of $E$ and $F$ and exploiting the second estimate in (2.1).

Recall from [28, Theorem 1.1] that we can rewrite Finsler area for $\mathscr{N}=\mathbb{R}^{m+1}$ as follows.

Theorem 2.2. If $\mathscr{N}=\mathbb{R}^{m+1}$ with a Finsler structure $F=F(x, y)$, then the Finsler area of any $C^{1}$-immersion $X$ of a smooth m-dimensional manifold $\mathscr{M}$ into $\mathbb{R}^{m+1}$ may be expressed in local coordinates $\left(u^{1}, \ldots, u^{m}\right): \Omega \subset \mathscr{M} \rightarrow \tilde{\Omega} \subset \mathbb{R}^{m}$ as

$$
\operatorname{area}_{\Omega}^{F}(X)=\int_{\tilde{\Omega}} \mathcal{A}^{F}\left(X(u),\left(\frac{\partial X}{\partial u^{1}} \wedge \ldots \wedge \frac{\partial X}{\partial u^{m}}\right)(u)\right) d u^{1} \wedge \ldots \wedge d u^{m},
$$

where

$$
\mathcal{A}^{F}(x, Z)=\frac{|Z| \mathscr{H}^{m}\left(B_{1}^{m}(0)\right)}{\mathscr{H}^{m}\left(\left\{T \in Z^{\perp} \subset \mathbb{R}^{m+1}: F(x, T) \leq 1\right\}\right)} \quad \text { for } \quad(x, Z) \in \mathbb{R}^{m+1} \times\left(\mathbb{R}^{m+1} \backslash\{0\}\right) .
$$


Notice that the explicit form (2.6) of the integrand $\mathcal{A}^{F}$ directly implies positive 1-homogeneity in its second argument:

$$
\mathcal{A}^{F}(x, t Z)=t \mathcal{A}^{F}(x, Z) \quad \text { for all }(x, Z) \in \mathbb{R}^{m+1} \times\left(\mathbb{R}^{m+1} \backslash\{0\}\right), t>0 .
$$

This together with the fact that $\mathcal{A}^{F}$ in the integral in (2.5) depends on the position $X(u)$ and on the normal vector $\left(X_{u^{1}} \wedge \ldots \wedge X_{u^{m}}\right)(u)$ qualifies $\mathcal{A}^{F}$ as Cartan integrand as defined in [25, p. 2]. Moreover, inspecting (2.6) one immediately sees that $\mathcal{A}^{F}$ does not depend on $x$ in case of a Minkowski metric $F=F(y)$, and $\mathcal{A}^{F}(x, Z)$ simplifies to the Euclidean area integrand $\mathcal{A}(Z)=|Z|$ if $F$ happens to be the Euclidean metric, $F(x, y)=E(y)=|y|$. Notice, in addition, that the Cartan integrand $\mathcal{A}^{F}$ is even in its second argument even if $F$ is not reversible. Finally, we will see in Corollary 2.10 below that $\mathcal{A}^{F}$ is smooth on $\mathbb{R}^{m+1} \times\left(\mathbb{R}^{m+1} \backslash\{0\}\right)$ since $F$ is smooth on this set.

Even if $F$ itself is not a Finsler metric one can deduce several useful properties of $\mathcal{A}^{F}$, for example, explicit $L^{\infty}$-bounds on $F=F(x, y)$ transfer to corresponding bounds on the Cartan area integrand $\mathcal{A}^{F}=\mathcal{A}(x, Z)$ proven in [28, Lemma 2.4]:

Lemma 2.3 (Pointwise bounds). Let $F_{1}(x, y), F_{2}(x, y)$ be continuous on $\mathbb{R}^{m+1} \times \mathbb{R}^{m+1}$, strictly positive for $y \neq 0$, and positively 1-homogeneous in the $y$-variable, and assume that for each $x \in$ $\mathbb{R}^{m+1}$ there exist numbers $0<c_{1}(x) \leq c_{2}(x)$ such that

$$
c_{1}(x) F_{1}(x, y) \leq F_{2}(x, y) \leq c_{2}(x) F_{1}(x, y) \quad \text { for all } y \in \mathbb{R}^{m+1},
$$

then the corresponding Cartan area integrands $\mathcal{A}^{F_{1}}$ and $\mathcal{A}^{F_{2}}$ satisfy

$$
c_{1}^{m}(x) \mathcal{A}^{F_{1}}(x, Z) \leq \mathcal{A}^{F_{2}}(x, Z) \leq c_{2}^{m}(x) \mathcal{A}^{F_{1}}(x, Z) \quad \text { for all } Z \in \mathbb{R}^{m+1} .
$$

If one wants to use this bridge between Finsler area and the variational theory for Cartan functionals to prove new results about Finsler-minimal immersions, one has to establish convexity of $\mathcal{A}^{F}$ in the $Z$-variable. For reversible Finsler metrics this was indeed proven geometrically by Busemann [6, Theorem II, p. 28] in the completely different context of volume computations for cross sections of convex bodies:

Theorem 2.4 (Busemann). If $F$ is a reversible Finsler metric on $\mathscr{N}=\mathbb{R}^{m+1}$, then the corresponding Cartan area integrand $\mathcal{A}^{F}=\mathcal{A}^{F}(x, Z)$ is convex in the $Z$-variable for any $x \in \mathbb{R}^{m+1}$.

It was Busemann's essential requirement of reversible Finsler metrics that motivated our choice of symmetrization for in general non-reversible Finsler structures $F$. That the $m$-harmonic symmetrization defined in (1.3) is indeed suitable is partially justified by the following result shown in [28, Lemma 2.3]:

Lemma 2.5. If $F=F(x, y)$ is continuous on $\mathbb{R}^{m+1} \times \mathbb{R}^{m+1}$, strictly positive whenever $y \neq 0$, and positively homogeneous in the $y$-variable, then

$$
\mathcal{A}^{F}(x, Z)=\mathcal{A}^{F_{\text {sym }}}(x, Z) \quad \text { for all }(x, Z) \in \mathbb{R}^{m+1} \times \mathbb{R}^{m+1} .
$$

Let us mention here that the $m$-harmonic symmetrization $F_{\text {sym }}$ is the unique even and positively 1homogeneous function generating the same area integrand as in (2.10). This is due to the invertibility of the extended spherical Radon transform on even positively $(-m)$-homogeneous smooth functions on $\mathbb{R}^{m+1} \backslash\{0\}$; see Theorem 2.11 and Lemma 2.7 in Section 2.2.

Convexity together with the pointwise bounds stated in Lemma 2.3 suffices to apply the existence theory for Cartan functionals, which helped solving the Plateau problem in Finsler 3-space in [28]. 
But in order to access available results on critical immersions for Cartan functionals one needs strict convexity of $\mathcal{A}^{F}$ in the $Z$-variable in the sense of parametric ellipticity. This is the strongest from of convexity that one can expect due to the homogeneity in the $Z$-variable, as described in the next subsection. Let us point out that we do not see how to quantify Busemann's original geometric proof to obtain strict convexity of the Cartan area integrand, which is why we devised an alternative route via the spherical Radon transform.

\subsection{Radon transform and parametric ellipticity}

The spherical Radon transform ${ }^{3}$ [29] functions that are continuous on the unit sphere:

Definition 2.6 (Spherical Radon transform). The spherical Radon transform $\widehat{\mathcal{R}}[f]$ of a function $f \in$ $C^{0}\left(\mathbb{S}^{m}\right)$ is defined as

$$
\widehat{\mathcal{R}}[f](\zeta):=\frac{1}{\mathscr{H}^{m-1}\left(\mathbb{S}^{m-1}\right)} \int_{\mathbb{S}^{m} \cap \zeta^{\perp}} f(\omega) d \mathscr{H}^{m-1}(\omega) \quad \text { for } \zeta \in \mathbb{S}^{m} .
$$

In the context of integral geometry, geometric tomography, and convex analysis the spherical Radon transform has been used intensively; see, e.g., [2, 17, 20-22]. In our present setting it is useful to look at a suitable homogeneous extension of the Radon transform. We define

$$
\mathcal{R}[g](Z):=\frac{1}{|Z|} \widehat{\mathcal{R}}\left[\left.g\right|_{\mathbb{S}^{m}}\right]\left(\frac{Z}{|Z|}\right) \quad \text { for } g \in C^{0}\left(\mathbb{R}^{m+1} \backslash\{0\}\right), Z \in \mathbb{R}^{m+1} \backslash\{0\},
$$

which by definition is a (-1)-homogeneous function on $\mathbb{R}^{m+1} \backslash\{0\}$.

In [28, Section 3] we analyzed in detail this homogeneous extension and proved among other things that $\mathcal{R}$ is a bounded linear map from $C^{0}\left(\mathbb{R}^{m+1} \backslash\{0\}\right)$ to itself [28, Corollary 3.3], and that $\mathcal{R}[g]$ is of class $C^{k}$ if $g$ is $(-m)$-homogeneous and itself of class $C^{k}$, together with an explicit differentiation formula [28, Theorem 3.6], to produce quantitative sufficient criteria to guarantee higher regularity for the solutions of the Plateau problem in Finsler 3-space. Here, we will need this differentiation rule only once, and only for the first order derivative:

$$
Z_{\tau} \frac{\partial}{\partial Z_{\sigma}} \mathcal{R}[g](Z)=-\mathcal{R}\left[\frac{\partial}{\partial y^{\tau}}\left(y^{\sigma} g\right)\right](Z)
$$

for all $Z=\left(Z^{1}, \ldots, Z^{m+1}\right), y=\left(y^{1}, \ldots, y^{m+1}\right) \in \mathbb{R}^{m+1} \backslash\{0\}$, where we set $Z_{\tau}:=\delta_{\tau l} Z^{l}$.

The key observation to connect the Radon transformation to Finsler-minimal surfaces is, that one can rewrite the Cartan area integrand $\mathcal{A}^{F}$ in terms of the extended spherical Radon transform; see [28, Corollary 3.8]:

Lemma 2.7 (Cartan area as Radon transform). For any function $F=F(x, y)$ that is continuous on $\mathbb{R}^{m+1} \times \mathbb{R}^{m+1}$, positive whenever $y \neq 0$, and positively homogeneous in the $y$-variable, one has the identity

$$
\mathcal{A}^{F}(x, Z)=\frac{1}{\mathcal{R}\left[F^{-m}(x, \cdot)\right](Z)} \quad \text { for }(x, Z) \in \mathbb{R}^{m+1} \times\left(\mathbb{R}^{m+1} \backslash\{0\}\right) .
$$

Our main goal in this section is to prove that every Finsler metric $F$ leads to a Cartan area integrand $\mathcal{A}^{F}(x, Z)$ that is strongly convex on $Z^{\perp}$ in the second variable, which corresponds to parametric ellipticity; see Definition 2.12 below. In order to do this we are going to use an indirect reasoning,

\footnotetext{
${ }^{3}$ For $m=2$ also known as the Funk transform [16].
} 
which requires to invert the Radon transform to go back and forth between the original Finsler metric $F$ and its image $\mathcal{A}^{F}$ under the Radon transform. Such an invertibility result is available only on the Fréchet space of even smooth functions on the sphere, since one gains some orders of derivatives under the Radon transform. In [20, Theorem 3.4.14 \& Proposition 3.6.4] one finds a quantitative estimate on the inverse proven by means of spherical harmonics that can be extended to all orders of differentiability; see [27, Prop. 2.2.26 \& Theorem 2.2.27]. But for our purposes it will suffice to prove continuity of the extended Radon transform $\mathcal{R}$ on a certain Fréchet subspace of $C^{\infty}\left(\mathbb{R}^{m+1} \backslash\{0\}\right)$, and to give an explicit formula of the inverse $\mathcal{T}:=\mathcal{R}^{-1}$ in terms of Helgason's inverse of the spherical Radon transform $\widehat{\mathcal{T}}=\widehat{\mathcal{R}}^{-1}$ as, e.g., presented in [21, Chapter III, Theorem 1.11]. Combining this with the open mapping theorem on Fréchet spaces yields the desired (non-quantitative) continuity of the inverse $\mathcal{T}$.

To begin with, recall from [28, Corollary 3.7] the following estimate of seminorms

$$
\varrho_{l}(g):=\max \left\{\left|D^{\alpha} g(\xi)\right|: \xi \in \mathbb{S}^{m},|\alpha| \leq l\right\} \quad \text { for } l=0,1, \ldots, k,
$$

on functions $g$ of class $C^{k}\left(\mathbb{R}^{m+1} \backslash\{0\}\right)$, where $\alpha=\left(\alpha_{1}, \ldots, \alpha_{m}\right)$ is a multi-index with $\alpha_{i} \in \mathbb{N} \cup\{0\}$ and with length $|\alpha|=\alpha_{1}+\cdots+\alpha_{m}$ :

Lemma 2.8. There is a constant $C=C(m, k)$ such that for any positively $(-m)$-homogeneous function $g \in C^{k}\left(\mathbb{R}^{m+1} \backslash\{0\}\right)$ one has

$$
\varrho_{k}(\mathcal{R}[g]) \leq C(m, k) \varrho_{k}(g)
$$

We can use (2.16) to estimate the seminorms

$$
p_{k}(f):=\max \left\{\left|D^{\alpha} f(Z)\right|: Z \in S_{k},|\alpha| \leq k\right\}, \quad k=0,1,2, \ldots,
$$

on the nested compact sets

$$
S_{k}:=\overline{B_{k+1}(0)} \backslash B_{\frac{1}{k+1}}(0) \subset \mathbb{R}^{m+1}
$$

for positively homogeneous functions:

Lemma 2.9. For a positively $q$-homogeneous function $f \in C^{k}\left(\mathbb{R}^{m+1} \backslash\{0\}\right)$ one has

$$
\varrho_{k}(f) \leq p_{k}(f) \leq(k+1)^{|q|+k} \varrho_{k}(f) \quad \text { for all } k=0,1,2, \ldots .
$$

Proof: The first estimate is trivial since $\mathbb{S}^{m} \subset S_{k}$ for all $k=0,1,2, \ldots$ Notice for the second inequality that $D^{\alpha} f$ is positively $(q-|\alpha|)$-homogeneous, i.e.,

$$
D^{\alpha} f(Z)=|Z|^{q-|\alpha|} D^{\alpha} f(Z /|Z|) \quad \text { for } Z \in \mathbb{R}^{m+1} \backslash\{0\},
$$

so that

$$
\begin{aligned}
\left|D^{\alpha} f(Z)\right| & \leq|Z|^{q-|\alpha|} \max \left\{\left|D^{\alpha} f(\xi)\right|: \xi \in \mathbb{S}^{m}\right\} \\
& \leq|Z|^{q-|\alpha|} \varrho_{k}(f) \quad \text { for }|\alpha| \leq k .
\end{aligned}
$$

For $Z \in S_{k}$ with $|Z| \geq 1$ one has $|Z|^{q-|\alpha|} \leq(k+1)^{|q|+|\alpha|}$, and for $1 /(k+1) \leq|Z|<1$ the estimate

$$
|Z|^{q-|\alpha|} \leq|Z|^{q}(k+1)^{|\alpha|}= \begin{cases}(k+1)^{|\alpha|}|Z|^{-|q|} & \text { for } q<0 \\ |Z|^{|q|}(k+1)^{|\alpha|} & \text { for } q>0\end{cases}
$$


and the right-hand side is clearly dominated by $(k+1)^{|q|+|\alpha|}$ as well. This leads to

$$
\left|D^{\alpha} f(Z)\right| \leq(k+1)^{|q|+k} \varrho_{k}(f) \quad \text { for all } Z \in S_{k},|\alpha| \leq k,
$$

for all $k=0,1,2, \ldots$, which concludes the proof.

Since $\mathbb{R}^{m+1} \backslash\{0\}=\bigcup_{k=1}^{\infty} S_{k}$, the space $C^{\infty}\left(\mathbb{R}^{m+1} \backslash\{0\}\right)$ equipped with the family of seminorms $p_{k}$ for $k=0,1,2, \ldots$, is a Fréchet space; see [31, Section 1.46]. The closed subspace of functions $g \in C^{\infty}\left(\mathbb{R}^{m+1} \backslash\{0\}\right)$ that are, in addition, positively $(-m)$-homogeneous is therefore itself a Fréchet space with respect to the same family of seminorms, and we can combine the preceding two lemmas to obtain the continuity of the extended Radon transform on this smaller Fréchet space.

Corollary 2.10 (Continuity of $\mathcal{R}$ ). The extended Radon transform $\mathcal{R}$ is a bounded linear mapping from the Fréchet space of $(-m)$-homogeneous mappings of class $C^{\infty}\left(\mathbb{R}^{m+1} \backslash\{0\}\right)$ into the Fréchet space of (-1)-homogenous mappings of class $C^{\infty}\left(\mathbb{R}^{m+1} \backslash\{0\}\right)$, satisfying the estimate

$$
p_{k}(\mathcal{R}[f]) \leq C(m, k)(k+1)^{k+1} p_{k}(f) \quad k=0,1,2, \ldots,
$$

where $f \in C^{\infty}\left(\mathbb{R}^{m+1} \backslash\{0\}\right)$ is an arbitrary positively $(-m)$-homogeneous function.

ProOF: That $\mathcal{R}$ is linear and that $\mathcal{R}[f]$ is positively $(-1)$-homogeneous can be seen directly from the definition (2.12). Hence we can apply (2.18) for $q:=-1$, and (2.16) for $g:=f$ to obtain

$$
\begin{aligned}
p_{k}(\mathcal{R}[f]) & \stackrel{(2.18)}{\leq} \\
& (k+1)^{1+k} \varrho_{k}(\mathcal{R}[f]) \\
& \stackrel{(2.16)}{\leq}(k+1)^{1+k} C(m, k) \varrho_{k}(f) \\
& (k+1)^{1+k} C(m, k) p_{k}(f) .
\end{aligned}
$$

Further restrictions to even smaller Fréchet spaces in the domain of $\mathcal{R}$ and in the target space are necessary to invert $\mathcal{R}$, since one can easily see that the kernel of $\mathcal{R}$ contains all odd functions.

Theorem 2.11 ( $\mathcal{R}$ as invertible mapping). The extended Radon transform $\mathcal{R}$ restricted to the Fréchet space of even and positively $(-m)$-homogeneous functions of class $C^{\infty}\left(\mathbb{R}^{m+1} \backslash\{0\}\right)$ is a continuous and bijective linear mapping onto the Fréchet space of even and positively (-1)-homogeneous smooth functions on $\mathbb{R}^{m+1} \backslash\{0\}$ ) with a continuous inverse $\mathcal{T}$.

Proof: The spherical Radon transform $\widehat{\mathcal{R}}$ is injective on the space of even continuous functions on the sphere $\mathbb{S}^{m}$; see [20, Proposition 3.4.12]. If $g_{1}, g_{2}$ are both, say $q$-homogeneous, even, and smooth on $\mathbb{R}^{m+1} \backslash\{0\}$, then the identity

$$
\mathcal{R}\left[g_{1}\right](Z)=\mathcal{R}\left[g_{2}\right](Z) \quad \text { for all } Z \in \mathbb{R}^{m+1} \backslash\{0\}
$$

implies by definition (see (2.12))

$$
\widehat{\mathcal{R}}\left[g_{1}{\mid \mathbb{S}^{m}}\right](Z /|Z|)=\widehat{\mathcal{R}}\left[\left.g_{2}\right|_{\mathbb{S}^{m}}\right](Z /|Z|) \quad \text { for all } Z \neq 0,
$$

from which we infer $\left.g_{1}\right|_{S^{m}}=\left.g_{2}\right|_{S^{m}}$ since the spherical Radon transform $\widehat{\mathcal{R}}$ is injective on such even functions. But then, by positive $q$-homogeneity,

$$
g_{1}(Z)=\left.|Z|{ }^{q} g_{1}\right|_{S^{m}}\left(\frac{Z}{|Z|}\right)=\left.|Z|^{q} g_{2}\right|_{S^{m}}\left(\frac{Z}{|Z|}\right)=g_{2}(Z) .
$$


In addition, Helgason presents in [21, Chapter III, Theorem 1.11] an explicit formula for the preimage $\widehat{\mathcal{T}}(\varphi)$ of any even function $\varphi$ of class $C^{\infty}\left(\mathbb{S}^{m}\right)$, which means that the spherical Radon transform is bijective on the space of even smooth functions on the sphere $\mathbb{S}^{m}$. Without using Helgason's explicit expression for this inverse we can use its existence to define for a positively $(-1)$-homogeneous and even function $\Phi \in C^{\infty}\left(\mathbb{R}^{m+1} \backslash\{0\}\right)$ the inverse $\mathcal{T}:=\mathcal{R}^{-1}$ of the extended Radon transformation $\mathcal{R}$ by

$$
\mathcal{T}[\Phi](y):=\frac{1}{|y|^{m}} \widehat{\mathcal{T}}\left[\left.\Phi\right|_{\mathbb{S} m}\right]\left(\frac{y}{|y|}\right) .
$$

Indeed, one calculates for an even positively $(-m)$-homogeneous function $f \in C^{\infty}\left(\mathbb{R}^{m+1} \backslash\{0\}\right)$, setting $\pi_{\mathbb{S} m}(y):=y /|y|$,

$$
\begin{aligned}
\mathcal{T}[\mathcal{R}[f]](y) & =\frac{1}{|y|^{m}} \widehat{\mathcal{T}}\left[\left.(\mathcal{R}[f])\right|_{\mathbb{S}^{m}}\right] \circ \pi_{\mathbb{S}^{m}}(y)=\frac{1}{|y|^{m}} \widehat{\mathcal{T}}\left[\left.\left(\left(|\cdot|^{-1} \widehat{\mathcal{R}}\left[\left.f\right|_{\mathbb{S}^{m}}\right]\right) \circ \pi_{\mathbb{S} m}\right)\right|_{\mathbb{S}^{m}}\right] \circ \pi_{\mathbb{S}^{m}}(y) \\
& =\frac{1}{|y|^{m}} \widehat{\mathcal{T}}\left[\left.\left(\widehat{\mathcal{R}}\left[\left.f\right|_{\mathbb{S}^{m}}\right] \circ \pi_{\mathbb{S}^{m}}\right)\right|_{\mathbb{S}^{m}}\right] \circ \pi_{\mathbb{S}^{m}}(y)=\frac{1}{|y|^{m}} \widehat{\mathcal{T}}\left[\left.\left(\widehat{\mathcal{R}}\left[\left.f\right|_{\mathbb{S}^{m}}\right]\right)\right|_{\mathbb{S}^{m}}\right] \circ \pi_{\mathbb{S}^{m}}(y) \\
& =\frac{1}{|y|^{m}} \widehat{\mathcal{T}}\left[\widehat{\mathcal{R}}\left[\left.f\right|_{\mathbb{S}^{m}}\right]\right] \circ \pi_{\mathbb{S}^{m}}(y)=\left.\frac{1}{|y|^{m}} f\right|_{\mathbb{S}^{m}} \circ \pi_{\mathbb{S}^{m}}(y)=\frac{1}{|y|^{m}} f(y /|y|)=f(y),
\end{aligned}
$$

where only in the very last equation we have used the positive $(-m)$-homogeneity of $f$. A similar calculation also shows that $\mathcal{R}[\mathcal{T}[\Phi]]=\Phi$ for all even, smooth, positively $(-1)$-homogeneous functions $\Phi$ on $\mathbb{R}^{m+1} \backslash\{0\}$. Since for any $q \in \mathbb{R}$ the space of even positively $q$-homogeneous functions of class $C^{\infty}\left(\mathbb{R}^{m+1} \backslash\{0\}\right)$ forms a closed linear subspace of the Fréchet space $C^{\infty}\left(\mathbb{R}^{m+1} \backslash\{0\}\right)$ with respect to the family of seminorms $p_{k}, k=0,1,2, \ldots$, we have established that $\mathcal{R}$ is a continuous (see Corollary 2.10), bijective linear mapping from the Fréchet space of even positively $(-m)$-homogeneous smooth mappings to the Fréchet space of even positively (-1)-homogeneous smooth mappings on $\mathbb{R}^{m+1} \backslash\{0\}$. Hence we can apply the open mapping theorem, in particular [31, 2.12 Corollaries (a), (b)], to obtain the continuity of $\mathcal{T}$.

Now we have collected all properties needed to prove the parametric ellipticity of the Cartan area integrand $\mathcal{A}^{F}$ generated by a Finsler metric $F=F(x, y)$. Let us recall this notion of convexity (see, e.g., [24, p. 298]) that is optimal for any Cartan integrand $\mathcal{C}=\mathcal{C}(x, Z)$ satisfying the homogeneity condition

$$
\mathcal{C}(x, t Z)=t \mathcal{C}(x, Z) \quad \text { for all }(x, Z) \in \mathbb{R}^{m+1} \times\left(\mathbb{R}^{m+1} \backslash\{0\}\right), t>0 .
$$

by virtue of Euler's relation $\mathcal{C}_{Z Z}(x, Z) Z=0$.

Definition 2.12 ((Parametric) ellipticity). A Cartan integrand $\mathcal{C}=\mathcal{C}(x, Z) \in C^{2}\left(\mathbb{R}^{m+1} \times \mathbb{R}^{m+1} \backslash\right.$ $\{0\})$ satisfying (2.21) is called (parametric) elliptic if and only if for every $R_{0}>0$ there is some number $\lambda_{\mathcal{C}}\left(R_{0}\right)>0$ such that the Hessian $\mathcal{C}_{Z Z}(x, Z)-\lambda_{\mathcal{C}}\left(R_{0}\right) \mathcal{A}_{Z Z}^{E}(Z)$ is positive semi-definite for all $(x, Z) \in \overline{B_{R_{0}}(0)} \times\left(\mathbb{R}^{m+1} \backslash\{0\}\right)$.

Recall from the remarks following Theorem 2.2 that we denoted the Euclidean metric by $E(y)=$ $|y|$, which explains the notation $\mathcal{A}^{E}$ for the Cartan area integrand generated by $E$, and we have noticed there that $\mathcal{A}^{E}(Z)=|Z|$.

Theorem 2.13 (Ellipticity). Let $F=F(x, y)$ be a reversible Finsler metric on $\mathbb{R}^{m+1}$. Then for each $x \in \mathbb{R}^{m+1}$ there exist constants $0<\lambda_{1}^{F}(x) \leq \lambda_{2}^{F}(x)$ such that

$$
\lambda_{1}^{F}(x) \xi \cdot \mathcal{A}_{Z Z}^{E}(Z) \xi \leq \xi \cdot \mathcal{A}_{Z Z}^{F}(x, Z) \xi \leq \lambda_{2}^{F}(x) \xi \cdot \mathcal{A}_{Z Z}^{E}(Z) \xi \quad \text { for all } \xi, Z \in \mathbb{R}^{m+1}, Z \neq 0 .
$$


The Hessian $\mathcal{A}_{Z Z}^{F}$ is $(-1)$-homogeneous in the $Z$-variable, and for the Euclidean metric one computes

$$
|Z| \xi \cdot \mathcal{A}_{Z Z}^{E}(Z) \xi=|\xi|^{2}-|Z|^{-2}(Z \cdot \xi)^{2}=\left|\pi_{Z^{\perp}}(\xi)\right|^{2},
$$

where $\pi_{Z^{\perp}}$ denotes the orthogonal projection onto the subspace $Z^{\perp}:=\left\{\eta \in \mathbb{R}^{m+1}: Z \cdot \eta=0\right\}$. Therefore we deduce from the left inequality in (2.22) by homogeneity

$$
\inf _{\substack{\bar{Z} \in \mathbb{S}^{m}, \bar{\eta} \in \mathbb{S}^{m} \cap \bar{Z} \perp}} \bar{\eta} \cdot \mathcal{A}_{Z Z}^{F}(x, \bar{Z}) \bar{\eta}=\inf _{\substack{Z \neq 0, \eta \in Z^{\perp} \backslash\{0\}}} \frac{|Z| \eta \cdot \mathcal{A}_{Z Z}^{F}(x, Z) \eta}{|\eta|^{2}} \geq \lambda_{1}^{F}(x)>0 .
$$

Since the right-hand side is positive for each $x \in \mathbb{R}^{m+1}$ according to Theorem 2.13, and the left-hand side is (Lipschitz) continuous in $x$, it attains its positive minimum $\lambda\left(R_{0}\right)>0$ on $\overline{B_{R_{0}}(0)} \subset \mathbb{R}^{m+1}$. This implies

$$
|Z| \eta \cdot \mathcal{A}_{Z Z}^{F}(x, Z) \eta \geq \lambda\left(R_{0}\right)|\eta|^{2} \quad \text { for all } Z \neq 0, x \in \overline{B_{R_{0}}(0)}, \eta \in Z^{\perp},
$$

which readily translates to

$$
|Z| \xi \cdot \mathcal{A}_{Z Z}^{F}(x, Z) \xi \geq \lambda\left(R_{0}\right)\left|\pi_{Z^{\perp}}(\xi)\right|^{2} \quad \text { for all } Z \neq 0, x \in \overline{B_{R_{0}}(0)}, \xi \in \mathbb{R}^{m+1} .
$$

Combining this with Lemma 2.5 we have shown that Theorem 2.13 implies the following:

Corollary 2.14 (Ellipticity). Let $F=F(x, y)$ be a Finsler metric on $\mathbb{R}^{m+1}$ satisfying assumption (GA). Then the corresponding Cartan area integrand $\mathcal{A}^{F}$ is (parametric) elliptic in the sense of Definition 2.12 .

Proof OF THEOREM 2.13: Fix $x \in \mathbb{R}^{m+1}$. We claim that the function

$$
Z \mapsto \mathcal{A}^{F}(x, Z)-\frac{1}{n}|Z|
$$

is convex for each $n \geq n_{0}$ if $n_{0}=n_{0}(x)$ is sufficiently large. From this statement the theorem follows.

To prove the claim we first apply Lemma 2.3 to the Finsler structures $F_{1}(x, y):=E(y)=|y|$ and $F_{2}(x, y):=F(x, y)$ to deduce from

$$
c_{1}(x) E(y):=\min _{\eta \in \mathbb{S}^{m}} F(x, \eta)|y| \leq F(x, y) \leq \max _{\eta \in \mathbb{S}^{m}} F(x, \eta)|y|=: c_{2}(x) E(y)
$$

the corresponding bounds on the Cartan area integrands $\mathcal{A}^{E}$ and $\mathcal{A}^{F}$,

$$
c_{1}^{m}(x) \mathcal{A}^{E}(Z) \leq \mathcal{A}^{F}(x, Z) \leq c_{2}^{m}(x) \mathcal{A}^{E}(Z) \quad \text { for all } Z \in \mathbb{R}^{m+1} .
$$

Notice that $0<c_{1}(x) \leq c_{2}(x)$ since $F$ as a Finsler metric with the properties (F1) and (F2) satisfies $F(x, y)>0$ for $y \neq 0$; see, e.g., [3, Theorem 1.2.2]. Thus, for all $n \in \mathbb{N}$ with $n \geq n_{1}(x)$, where $n_{1}(x)$ is the smallest integer greater or equal $c_{1}(x)^{-1}$, the expression

$$
\Phi_{n}(x, Z):=\mathcal{A}^{F}(x, Z)-\frac{1}{n}|Z|
$$

is an even, positively 1-homogenous smooth function on $\mathbb{R}^{m+1} \backslash\{0\}$ (recall that $x$ is fixed), with $\Phi_{n}(x, Z)>0$ for $Z \neq 0$; cf. Lemma 2.1 and our remarks directly following Theorem 2.2. Hence, by virtue of Theorem 2.11, the inverse $\mathcal{T}$ of the extended Radon transfrom $\mathcal{R}$ can be applied to $1 / \Phi_{n}(x, \cdot)$. 
Before doing so we use Lemma 2.7 to rewrite the function $\Phi_{n}$ as

$$
\Phi_{n}(x, Z)=\mathcal{A}^{F}(x, Z)-\frac{1}{n}|Z|=\frac{1}{\mathcal{R}\left[F(x, \cdot)^{-m}\right]}-\frac{1}{n} \frac{1}{\mathcal{R}\left[|\cdot|^{-m}\right]}=\frac{\mathcal{R}\left[|\cdot|^{-m}\right]-\frac{1}{n} \mathcal{R}\left[F(x, \cdot)^{-m}\right]}{\mathcal{R}\left[F(x, \cdot)^{-m}\right] \mathcal{R}\left[|\cdot|^{-m}\right]} .
$$

Applying $\mathcal{T}$ to the function $1 / \Phi_{n}(x, \cdot)$ (for fixed $x$ and $n \geq n_{1}(x)$ ) now yields

$$
\mathcal{T}\left(\frac{1}{\Phi_{n}(x, \cdot)}\right)=\mathcal{T}\left(\frac{\mathcal{R}\left[F^{-m}(x, \cdot)\right] \mathcal{R}\left[|\cdot|^{-m}\right]}{\mathcal{R}\left[|\cdot|^{-m}\right]-\frac{1}{n} \mathcal{R}\left[F^{-m}(x, \cdot)\right]}\right) .
$$

The argument of $\mathcal{T}$ on the right-hand side is an even (-1)-homogenous function that tends to the expression $\mathcal{R}\left[F^{-m}(x, \cdot)\right]$ as $n \rightarrow \infty$ in the Fréchet space of even $(-1)$-homogeneous smooth functions on $\mathbb{R}^{m+1} \backslash\{0\}$ with respect to the topology given by the family of seminorms $p_{k}, k=0,1,2, \ldots$, introduced in (2.17). By the continuity of $\mathcal{T}$ with respect to that convergence (granted by Theorem 2.11) we find that

$$
\Psi_{n}(x, \cdot):=\left[\mathcal{T}\left(\frac{1}{\Phi_{n}(x, \cdot)}\right)\right]^{-1 / m} \longrightarrow F(x, \cdot) \text { as } n \rightarrow \infty
$$

in the Fréchet space of even 1-homogeneous smooth functions on $\mathbb{R}^{m+1} \backslash\{0\}$ with respect to the family of seminorms $p_{k}, k=0,1,2 \ldots$. (Notice that the left-hand side of (2.25) is even and positively $(-m)$-homogeneous according to Theorem 2.11, so that the left-hand side of (2.26) is even and positively 1-homogeneous.)

$F$ itself is a reversible Finsler metric satisfying (F1) and (F2) by assumption. In particular, for the positively 0 -homogeneous Hessian $\left(F^{2} / 2\right)_{y y}(x, y)$ one finds for fixed $x$ a constant $\Lambda_{1}^{F}(x)>0$ such that

$$
\xi \cdot\left(\frac{F^{2}}{2}\right)_{y y}(x, y) \xi \geq \Lambda_{1}^{F}(x)|\xi|^{2} \quad \text { for all } \xi \in \mathbb{R}^{m+1}, y \neq 0 .
$$

By virtue of the convergence in (2.26) there is some $n_{0}(x) \geq n_{1}(x)$ such that

$$
\xi \cdot \Psi_{n}(x, y) \xi \geq \frac{\Lambda_{1}^{F}}{2}|\xi|^{2} \quad \text { for all } n \geq n_{0}(x), \xi \in \mathbb{R}^{m+1}, y \neq 0
$$

which qualifies

$$
\Psi_{n}(x, \cdot)=\left[\mathcal{T}\left(\frac{1}{\Phi_{n}(x, \cdot)}\right)\right]^{-1 / m}
$$

as a reversible Finsler metric on $\mathbb{R}^{m+1}$ for each $n \geq n_{0}(x)$. Consequently, by Busemann's convexity result, Theorem 2.4, the corresponding Cartan area integrand $\mathcal{A}^{\Psi_{n}(x, \cdot)}=\mathcal{A}^{\Psi_{n}(x, \cdot)}(x, Z)$ is convex in the $Z$-variable. By means of Lemma 2.7 we can rewrite this Cartan area integrand as

$$
\mathcal{A}^{\Psi_{n}(x, \cdot)}(x, Z)=\frac{1}{\mathcal{R}\left[\Psi_{n}^{-m}(x, \cdot)\right](Z)}=\frac{1}{\mathcal{R}\left[\mathcal{T}\left(\frac{1}{\Phi_{n}(x, \cdot)}\right)\right]}=\Phi_{n}(x, Z)=\mathcal{A}^{F}(x, Z)-\frac{1}{n}|Z|,
$$

which establishes the left inequality in (2.22) for $\lambda_{1}^{F}(x)=1 / n_{0}(x)$. The right inequality in (2.22) simply follows from the fact that $\mathcal{A}^{F}(x, Z)$ is smooth on $\mathbb{R}^{m+1} \backslash\{0\}$ and positively 1-homogeneous. 


\section{Proofs of the main results}

Finsler-minimal graphs. First, we will combine our results of Section 2 with well-known results of Jenkins [26], L. Simon [34], and Winklmann [40] on solutions of the non-parametric Euler-Lagrange equations of elliptic Cartan functionals (whose integrand does not depend explicitly on the position vector), to prove the Bernstein result.

Proof of Theorem 1.1: Let $F=F(y)$ be a Minkowski metric on $\mathbb{R}^{m+1}$. Any entire Finslerminimal graph $\left\{(u, f(u)): u \in \mathbb{R}^{m}\right\}$ is by definition a critical immersion of Finsler area, which according to Theorem 2.2 - can be written as the variational integral

$$
\int_{\mathbb{R}^{m}} \mathcal{A}^{F}\left(\left(X_{u^{1}} \wedge \ldots \wedge X_{u^{m}}\right)(u)\right) d u^{1} \wedge \ldots \wedge d u^{m}
$$

where $X(u)=(u, f(u))$ for $u \in \mathbb{R}^{m}$. Here, the Cartan area integrand $\mathcal{A}^{F}=\mathcal{A}^{F}(Z)$ does not depend on the position vector, is smooth on $\mathbb{R}^{m+1} \backslash\{0\}$ and positively 1-homogeneous (see (2.7) and our remarks directly following Theorem 2.2). In addition, since $F=F(y)$ does not depend on the $x$-variable, we have the simple estimate (cf. (1.6))

$$
m_{F}|y|=\min _{\eta \in \mathbb{S}^{m}} F(\eta)|y| \leq F(y /|y|)|y| \leq \max _{\zeta \in \mathbb{S}^{m}} F(\zeta)|y|=M_{F}|y|
$$

with $0<m_{F} \leq M_{F}$ by the defining properties (F1) and (F2) which together imply that $F(y)>0$ as long as $y \neq 0$. Lemma 2.3 implies that

$$
m_{F}^{m}|Z| \leq \mathcal{A}^{F}(Z) \leq M_{F}^{m}|Z|
$$

By virtue of the general assumption (GA) and Corollary 2.14 we know that the Cartan area integrand $\mathcal{A}^{F}=\mathcal{A}^{F}(Z)$ is (parametric) elliptic. Since there is no $x$-dependence here, we therefore have the simplified ellipticity condition (cf. (2.23))

$$
|Z| \xi \cdot \mathcal{A}_{Z Z}^{F}(Z) \xi \geq \lambda\left|\pi_{Z^{\perp}}(\xi)\right|^{2}
$$

for some positive constant $\lambda$.

By scaling we may assume that $\mu:=\min \left\{m_{F}, \lambda\right\} \geq 1$, since a critical immersion $X$ for (3.1) is also critical for this integral with $\mathcal{A}^{F}$ replaced by $\mu^{-1} \mathcal{A}^{F}$.

Thus we have verified that the general assumptions (i)-(iii) on the Cartan integrand in [34, p. 266] (or alternatively without the need to scale in [26, p. 181]) are satisfied. The non-parametric EulerLagrange equation is given, e.g., in [34, formula (1)], and the function $f \in C^{2}\left(\mathbb{R}^{m}\right)$ generating the entire Finsler-minimal graph $\left\{(u, f(u)): u \in \mathbb{R}^{m}\right\}$ solves that equation on $\mathbb{R}^{m}$.

The Bernstein theorem for $m=2$ formulated in part (i) now follows either from [26, Theorem $3 \&$ Corollary] or from the first statement in [34, Corollary 1]. (Notice for the latter that Simon introduces the class $\mathscr{M}^{\prime}$ of generalized surfaces that can be represented as a graph of a $C^{2}$-function, and this class is contained in the class $\mathscr{M}$ of hypersurfaces mentioned in the first statement of his Corollary 1.) For $m=3$ we refer to the explicit statement regarding entire solutions of the non-parametric Euler-Lagrange equation in the second part of [34, Corollary 1].

For the proof of part (ii) of Theorem 1.1 we recall the seminorms $\varrho_{k}$ on the sphere introduced in (2.15), and assume to the contrary that for a sequence of Minkowski norms $F_{n}=F_{n}(y)$ on $\mathbb{R}^{m+1}$ converging up to third order to the Euclidean metric $E(y)=|y|$,

$$
\varrho_{3}\left(F_{n}-E\right) \longrightarrow 0 \text { as } n \rightarrow \infty,
$$


we have entire Finsler-minimal graphs $X_{n}(u):=\left(u, f_{n}(u)\right)$ that are not affine planes. Here, $X_{n}$ is critical for the Finsler area area $\mathbb{F}_{\mathbb{R}_{n}}$. From (3.5) we deduce, in particular, by means of the triangle inequality, that there is $n_{0} \in \mathbb{N}$ such that

$$
\frac{1}{2}=\frac{1}{2} \varrho_{0}(E) \leq \varrho_{0}\left(F_{n}\right) \leq 2 \varrho_{0}(E)=2 \quad \text { for all } n \geq n_{0} .
$$

Hence, by the chain rule and by (3.5),

$$
\varrho_{3}\left(F_{n}^{-m}-E^{-m}\right) \longrightarrow 0 \quad \text { as } n \rightarrow \infty,
$$

which according to Lemma 2.8 implies for the (linear) extended Radon transformation $\mathcal{R}$ :

$$
\varrho_{3}\left(\mathcal{R}\left[F_{n}^{-m}\right]-\mathcal{R}\left[E^{-m}\right]\right)=\varrho_{3}\left(\mathcal{R}\left[F_{n}^{-m}-E^{-m}\right]\right) \longrightarrow 0 \quad \text { as } n \rightarrow \infty .
$$

Again, by the triangle inequality,

$$
\frac{1}{2} \leq C_{1}(m):=\frac{1}{2} \varrho_{3}\left(\mathcal{R}\left[E^{-m}\right]\right) \leq \varrho_{3}\left(\mathcal{R}\left[F_{n}^{-m}\right]\right) \leq 2 \varrho_{3}\left(\mathcal{R}\left[E^{-m}\right]\right)=: C_{2}(m) \text { for all } n \geq n_{1}
$$

for some $n_{0} \leq n_{1} \in \mathbb{N}$. Notice that

$$
2 C_{1}(m) \geq \varrho_{0}\left(\mathcal{R}\left[E^{-m}\right]\right)=\max \left\{\left(\mathcal{A}^{E}(Z)\right)^{-1}=|Z|^{-1}, Z \in \mathbb{S}^{m}\right\}=1,
$$

where we used Lemma 2.7. By the same lemma, on the other hand, for $Z \in \mathbb{R}^{m+1} \backslash\{0\}$,

$$
\mathcal{A}^{F_{n}}(Z)-\mathcal{A}^{E}(Z) \stackrel{(2.14)}{=} \frac{1}{\mathcal{R}\left[F_{n}^{-m}\right](Z)}-\frac{1}{\mathcal{R}\left[|\cdot|^{-m}\right](Z)}=\frac{\mathcal{R}\left[|\cdot|^{-m}\right](Z)-\mathcal{R}\left[F_{n}^{-m}\right](Z)}{\mathcal{R}\left[F_{n}^{-m}\right](Z) \mathcal{R}\left[|\cdot|^{-m}\right](Z)} .
$$

Taking the $\varrho_{3}$-seminorm of this expression with a careful application of the Leibniz product rule, one can then use (3.8) to find a constant $C_{3}(m) \geq 0$ such that

$$
\varrho_{3}\left(\mathcal{A}^{F_{n}}(Z)-\mathcal{A}^{E}(Z)\right) \leq C_{3}(m) \varrho_{3}\left(\mathcal{R}\left[|\cdot|^{-m}\right]-\mathcal{R}\left[F_{n}^{-m}\right]\right) \quad \text { for all } n \geq n_{1},
$$

and the right-hand side converges to zero as $n \rightarrow \infty$ by virtue of (3.7). Thus, for any given $\eta>0$ there is $n_{2} \in \mathbb{N}$ with $n_{2} \geq n_{1}$, such that

$$
\sum_{|\alpha| \leq 3}\left|D^{\alpha}\left(\mathcal{A}^{F_{n}}-\mathcal{A}^{E}\right)(\xi)\right|<\eta \quad \text { for all } \xi \in \mathbb{S}^{m}, n \geq n_{2},
$$

which is exactly the condition that Simon requires for his Bernstein result for solutions of the nonparametric Euler-Lagrange equations of (3.1) in dimensions $m \leq 7$ ( [34, Corollary $2 \&$ (6)]. Hence, all Finsler-minimal graphs $X_{n}$, for $n \geq n_{2}$, are affine planes, contradicting our assumption.

It remains to prove part (iii) of Theorem 1.1. For this we verify the conditions Winklmann assumes for his Bernstein result [40, Theorem 4.1] for $C^{2}$-solutions of the non-parametric Euler-Lagrange equation. Indeed, the present Cartan area integrand $\mathcal{A}^{F}=\mathcal{A}^{F}(Z)$ satisfies the positive 1-homogeneity and ellipticity condition required in [40, (2.1) \& (2.2)] (see (2.7) and (3.4) in our context), and the $C^{3}$ vicinity quantized with a constant $\delta_{\star}(m, \gamma)$ in [40, p. 383] translates to condition (1.4) by means of the indirect argument presented for the proof of part (ii), where the final contradiction will be obtained by [40, Theorem 4.1] in this case, which concludes the proof. 
The uniqueness result for Finsler-minimal graphs, Theorem 1.2, will be established connecting our results of Section 2 to a more recent weighted energy estimate of Hildebrandt and Sauvigny [23, Theorem 3.1].

PROOF OF THEOREM 1.2: In the proof of Theorem 1.1 we have established already that both Finsler-minimal graphs $f_{1}, f_{2} \in C^{0}(\bar{\Omega} \backslash K) \cap C^{2}(\Omega \backslash K)$ are solutions of the non-parametric EulerLagrange equation of the Cartan functional (3.1) on the domain $\Omega \backslash K \subset \mathbb{R}^{m}$. The ellipticity condition (3.4) is a simpler version of the ellipticity condition [23, (3.7)] since we have no explicit dependence on the position vector in the Cartan area integrand in (3.1). This translates into an ellipticity condition of the non-parametric functional (where we can ignore the $x$ - and $z$-dependence in [23, (3.14)]), which is explicitly assumed in [23, Theorem 3.1]. Hence we can apply this theorem in the simpler situation of the homogeneous non-parametric Euler-Lagrange equation, i.e., for the right-hand side $H=H(x, z) \equiv 0$ in [23, (3.22)]. The resulting weighted energy estimate of Hildebrandt and Sauvigny reads therefore in our situation as follows:

$$
\int_{\Omega \backslash K} \mu\left(f_{1}, f_{2}\right)\left|\nabla f_{1}-\nabla f_{2}\right|^{2} d x \leq \frac{2}{\lambda} \int_{\partial \Omega}\left|f_{1}-f_{2}\right| d \mathscr{H}^{m-1},
$$

where $\mu\left(f_{1}, f_{2}\right)(u):=\left(\max \left\{\sqrt{1+\nabla f_{1}^{2}(u)}, \sqrt{1+\nabla f_{2}^{2}(u)}\right\}\right)^{-3}$ for $u \in \Omega \backslash K$. Now we can proceed as in the proof of [23, Theorem 4.1]. Assuming that $\left.f_{1}\right|_{\partial \Omega}=\left.f_{2}\right|_{\partial \Omega}$ we conclude $\nabla f_{1} \equiv \nabla f_{2}$ on $\Omega \backslash K$, and since $\Omega \backslash K$ is a domain this implies that $f_{1}-f_{2} \equiv$ const on $\Omega \backslash K$. But $f_{1}-f_{2}$ is of class $C^{0}(\bar{\Omega} \backslash K)$ and $K$ is compactly contained in $\Omega$, so that $f_{1} \equiv f_{2}$ on $\partial \Omega$ leads to equality of $f_{1}$ and $f_{2}$ on all of $\bar{\Omega} \backslash K$.

Uniqueness results like Theorem 1.2 can be used to extend solutions $f_{1}$ of certain partial differential equations on domains $\Omega \backslash K$ to all of $\Omega$, if the Dirichlet problem on $\Omega$ with prescribed boundary data $f_{1}$ on $\partial \Omega$ can be solved by a function, say $f_{2}$. The uniqueness theorem then implies that this solution $f_{2}$ coincides with $f_{1}$ on $\Omega \backslash K$, so that the original singular set $K$ of $f_{1}$ is removed by extending $f_{1}$ to the solution $f_{2}$ on all of $\Omega$. Such a result can be found in [23, Remark 4.7] but under slightly stronger assumptions on the singular set $K$, so we will draw from L. Simon's contribution [33] to prove Theorem 1.3.

Proof OF THEOREM 1.3. As in the proof of Theorem 1.1 we find that any Finsler-minimal graph $\{(u, f(u)): u \in \Omega \backslash K\}$ of class $C^{2}$ in Finsler-Minkowski space $\left(\mathbb{R}^{m+1}, F=F(y)\right)$ is generated by a function $f \in C^{2}(\Omega \backslash K)$ that solves the non-parametric Euler-Lagrange equation for the variational integral (3.1) as, e.g., presented in [33, formula (5)]. Also the general assumptions [33, formulae (1)-(4)] are satisfied in our present situation (cf. (3.3) and (3.4), modulo the simple scaling argument mentioned in the proof of Theorem 1.1 to obtain $\mu:=\min \left\{m_{F}, \lambda\right\} \geq 1$ for the exact factors in inequalities [33, (2) \& (3)]). Now, Theorem 1.3 follows from [33, Theorem 1].

Global results for Finsler-minimal immersions. Combining our results of Section 2 with global results on critical immersions of Cartan functionals by Clarenz et. al.,and Winklmann [9, 11, 38, 39] we establish in the following the convex hull property formulated in Theorem 1.4, the isoperimetric inequalities in Theorem 1.5, and the existence results for Finsler-minimal immersions in Theorem 1.6.

Proof of TheOrem 1.4. If $F=F(y)$ is a Minkowski metric on $\mathbb{R}^{m+1}$ then $F^{-m}$ is smooth on $\mathbb{R}^{m+1} \backslash\{0\}$ by virtue of (3.2), so that Lemma 2.7 in combination with Corollary 2.10 leads to a Cartan area integrand $\mathcal{A}^{F}=\mathcal{A}^{F}(Z)$ that is positively 1-homogeneous and smooth on $\mathbb{R}^{m+1} \backslash\{0\}$. In addition, we use the general assumption (GA) together with Corollary 2.14 as in the proof of Theorem 1.1 to 
show that $\mathcal{A}^{F}$ is (parametric) elliptic with the uniform estimate (3.4), which coincides with the ellipticity condition used in $\left[9\right.$, Definition 2.2]. Since we assume that $X \in C^{2}\left(\mathscr{M}, \mathbb{R}^{m+1}\right) \cap C^{0}\left(\overline{\mathscr{M}}, \mathbb{R}^{m+1}\right)$ is a Finsler-minimal immersion we know that $X$ is critical for the Cartan area functional (3.1); in the language of Clarenz [9] $X$ is extremal for (3.1). This implies by [9, Theorem 1] that its $\mathcal{A}^{F}$-mean curvature $H_{\mathcal{A}^{F}}$ vanishes since the Cartan area integrand $\mathcal{A}^{F}$ does not depend on the position vector. Hence all conditions of [9, Theorem 2.3] are satisfied which establishes the convex hull property for $X$.

The first variation formula for general Cartan functionals derived by Räwer [30] and Clarenz [8] were used by Clarenz et al. and Winklmann to derive various isoperimetric inequalities for critical immersions of such functionals $[10,11,38]$. Some of these results are used in the present context in connection with Section 2 to derive corresponding results for Finsler-minimal immersions.

Proof OF THEOREM 1.5. The first two parts of this theorem deal with Finsler-minimal immersions in Minkowski space. The Cartan area integrand $\mathcal{A}^{F}$ derived from the Minkowski metric $F=F(y)$ depends only on the $Z$-variable, and such Cartan functionals without dependence on the position vector have been considered by Winklmann in [38]. Notice that Winklmann does not use positivity of his Cartan integrand, although we have it here for $\mathcal{A}^{F}(Z)$ automatically by means of (3.3). Now Winklmann's isoperimetric inequality in [38, Corollary 2.3$]$ reads in our context as

$$
\operatorname{area}_{\mathscr{M}}^{F}(X) \leq \frac{1}{m} \frac{R}{m_{F}}\left\|\mathcal{A}_{Z}^{F}\right\|_{C^{0}\left(\mathbb{S}^{m}, \mathbb{R}^{m+1}\right)} \int_{\partial \mathscr{M}} d S
$$

Recall that $R>0$ is the radius of the closed Finsler ball $\overline{B_{R}^{F}(0)} \subset \mathbb{R}^{m+1}$ containing the boundary $X(\partial \mathscr{M})$, and therefore by the convex hull property, Theorem 1.4, also $X(\overline{\mathscr{M}})$. Notice further, that $\overline{B_{R}^{F}(0)} \subset \overline{B_{R / m_{F}}(0)}$, which can be shown by an argument similar to the one leading to (2.3) in the proof of Lemma 2.1. To estimate the supremum-norm of $\mathcal{A}_{Z}^{F}$ purely in terms of quantities explicitly given by the Minkowski metric $F$ we use Lemma 2.7 and the differentiation rule for the Radon transform in the simple form (2.13) (contracted by multiplication with $Z^{\tau}$ and summation over $\tau$ to compute in a first step for $Z \in \mathbb{S}^{m}$

$$
\begin{aligned}
\mathcal{A}_{Z_{\sigma}}^{F}(Z) \stackrel{(2.14)}{=}\left(\frac{1}{\mathcal{R}\left[F^{-m}\right]}\right)_{Z_{\sigma}}(Z)=-\frac{\left(\mathcal{R}\left[F^{-m}\right]\right)_{Z_{\sigma}}(Z)}{\mathcal{R}^{2}\left[F^{-m}\right](Z)} \\
\stackrel{(2.13)}{=} \frac{1}{\mathcal{R}^{2}\left[F^{-m}\right](Z)} \sum_{\tau=1}^{m+1} \mathcal{R}\left[Z^{\tau} \frac{\partial}{\partial y^{\tau}}\left(y^{\sigma} F^{-m}\right)\right](Z) \\
=\frac{1}{\mathcal{R}^{2}\left[F^{-m}\right](Z)} \sum_{\tau=1}^{m+1} \mathcal{R}\left[Z^{\tau}\left(\delta_{\tau}^{\sigma} F^{-m}-m y^{\sigma} F^{-m-1} F_{y^{\tau}}\right)\right](Z) \\
=\frac{Z^{\sigma}}{\mathcal{R}\left[F^{-m}\right](Z)}-\frac{m}{\mathcal{R}^{2}\left[F^{-m}\right](Z)} \sum_{\tau=1}^{m+1} \mathcal{R}\left[Z^{\tau} y^{\sigma} F^{-m-1} F_{y^{\tau}}\right](Z),
\end{aligned}
$$

where we used the linearity of the Radon several times. Therefore, we obtain for the norm of the 
gradient $\mathcal{A}_{Z}^{F}(Z)$ at $Z \in \mathbb{S}^{m}$ (again using linearity of $\mathcal{R}$ )

$$
\begin{aligned}
\left|\mathcal{A}_{Z}^{F}(Z)\right|^{2}= & \frac{1}{\mathcal{R}^{2}\left[F^{-m}\right](Z)}-\frac{2 m}{\mathcal{R}^{3}\left[F^{-m}\right](Z)} \sum_{\sigma, \tau=1}^{m+1} \mathcal{R}\left[Z^{\tau} Z^{\sigma} y^{\sigma} F^{-m-1} F_{y \tau}\right](Z) \\
& +\frac{m^{2}}{\mathcal{R}^{4}\left[F^{-m}\right](Z)} \sum_{\sigma=1}^{m+1}\left(\sum_{\tau=1}^{m+1} \mathcal{R}\left[Z^{\tau} y^{\sigma} F^{-m-1} F_{y^{\tau}}\right](Z) \sum_{\rho=1}^{m+1} \mathcal{R}\left[Z^{\rho} y^{\sigma} F^{-m-1} F_{y^{\rho}}\right](Z)\right)
\end{aligned}
$$

Since the spherical Radon transform $\widehat{\mathcal{R}}$ coincides with the extended Radon transform $\mathcal{R}$ on the sphere $\mathbb{S}^{m}$ we can use the explicit formula (2.11) to show that the second term on the right-hand side above vanishes: for $g(y):=\sum_{\tau=1}^{m+1} Z^{\tau} F^{-m-1}(y) F_{y^{\tau}}(y)$ we compute for a fixed $Z \in \mathbb{S}^{m}$ by linearity of $\mathcal{R}$

$$
\sum_{\sigma=1}^{m+1} \mathcal{R}\left[Z^{\sigma} y^{\sigma} g(\cdot)\right](Z)=\mathcal{R}[(Z \cdot y) g(\cdot)] \stackrel{(2.11)}{=} \frac{1}{\mathscr{H}^{m-1}\left(\mathbb{S}^{m-1}\right)} \int_{y \in \mathbb{S}^{m} \cap Z^{\perp}}(Z \cdot y) g(y) d \mathscr{H}^{m-1}(y)=0 .
$$

Consequently, it suffices to estimate the first and the last term on the right-hand side of (3.12) in terms of quantities related to the Minkowski metric $F$. By Lemma 2.7 we immediately see that the first term coincides with $\left(\mathcal{A}^{F}(Z)\right)^{2}$. For the last term in (3.12) we set $h(y):=\left(Z \cdot F_{y}(y)\right) / F(y)$ and use again the explicit integral formula (2.11) to estimate for fixed $\sigma \in\{1, \ldots, m+1\}$

$$
\begin{aligned}
\sum_{\tau=1}^{m+1} \mathcal{R}\left[Z^{\tau} y^{\sigma} F^{-m-1} F_{y^{\tau}}\right](Z) & =\mathcal{R}\left[y^{\sigma}\left(Z \cdot F_{y}(y)\right) / F(y) F^{-m}(y)\right] \\
& =\frac{1}{\mathscr{H}^{m-1}\left(\mathbb{S}^{m-1}\right)} \int_{y \in S^{m} \cap Z^{\perp}} y^{\sigma} h(y) F^{-m}(y) d \mathscr{H}^{m-1}(y) \\
& \leq \frac{\max _{y \in S^{m} \cap Z^{\perp}}|h(y)|}{\mathscr{H}^{m-1}\left(\mathbb{S}^{m-1}\right)} \int_{y \in S^{m} \cap Z^{\perp}} F^{-m}(y) d \mathscr{H}^{m-1}(y) \\
& =\left(\max _{y \in S^{m} \cap Z^{\perp}}|h(y)|\right) \mathcal{R}\left[F^{-m}\right],
\end{aligned}
$$

so that we deduce from (3.12) in connection with (3.3) the gradient estimate for the Cartan area integrand at $Z \in S^{m}$

$\left|\mathcal{A}_{Z}^{F}(Z)\right|^{2} \leq\left(\mathcal{A}^{F}(Z)\right)^{2}\left(1+m^{2} \sum_{\sigma=1}^{m+1}\left(\max _{y \in S^{m} \cap Z^{\perp}}|h(y)|^{2}\right)\right) \leq M_{F}^{2 m}\left(1+(m+1) m^{2} \max _{y \in S^{m} \cap Z^{\perp}}|h(y)|^{2}\right)$.

To estimate $|h(y)|$ for $y \in S^{m} \cap Z^{\perp}$ recall that the fundamental tensor $\left(g_{i j}\right)(y)=\left(\left(F^{2} / 2\right)_{y^{i} y^{j}}\right)(y)$ is positive definite by (F2). Combining this with the homogeneity (F1) one can easily deduce that the Hessian $F_{y^{i} y^{j}}(y)$ is positive semidefinite; see, e.g., formula (1.2.9) in [3, Proof of Theorem 1.2.2]. Thus,

$$
\Lambda(F)\left|F_{y}(y)\right|^{2} \geq F_{y^{i}}(y)\left(\frac{F^{2}}{2}\right)_{y^{i} y^{j}}(y) F_{y^{j}}(y)=F_{y^{i}}(y)\left(F_{y^{i}} F_{y^{j}}+F F_{y^{i} y^{j}}\right)(y) F_{y^{j}}(y) \geq\left|F_{y}(y)\right|^{4}
$$

for every $y \in \mathbb{S}^{m}$, where

$$
\Lambda(F):=\max _{\eta, \zeta \in \mathbb{S}^{m}} \eta \cdot\left(F^{2} / 2\right)_{y y}(\zeta) \eta
$$


is the largest possible eigenvalue of the fundamental tensor $g_{i j}$ of $F$ when restricted to the sphere $\mathbb{S}^{m}$. (Recall the summation convention from our introduction described under (F2).) Since $F$ is bounded from below by $m_{F}>0$ on $\mathbb{S}^{m}$ (see (3.2)), we obtain

$$
|h(y)|^{2} \leq \frac{1}{m_{F}^{2}}|Z|^{2} \Lambda(F) \quad \text { for all } y \in \mathbb{S}^{m} .
$$

Inserting this into (3.13) gives

$$
\left|\mathcal{A}_{Z}^{F}(Z)\right| \leq M_{F}^{m} \sqrt{1+\Lambda(F)(m+1) \frac{m^{2}}{m_{F}^{2}}} \quad \text { for all } Z \in \mathbb{S}^{m},
$$

which according to (3.11) leads to the following isoperimetric inequality

$$
\operatorname{area}_{\mathscr{M}}^{F}(X) \leq \frac{R}{m} \frac{M_{F}^{m}}{m_{F}} \sqrt{1+\Lambda(F)(m+1) \frac{m^{2}}{m_{F}^{2}}} \int_{\partial \mathscr{M}} d S .
$$

Recall from our remarks preceding Theorem 1.5 that $\int_{\partial \mathscr{M}} d S_{F}=\operatorname{area}_{\partial \mathscr{M}}^{F}\left(\left.X\right|_{\partial \mathscr{M}}\right)$ which specializes to $\int_{\partial \mathscr{M}} d S=\operatorname{area}_{\partial \mathscr{M}}^{E}\left(\left.X\right|_{\partial \mathscr{M}}\right)$ if the Finsler metric $F$ happens to be Euclidean, i.e., $F(x, y)=E(y)=$ $|y|$. Now, Lemma 2.1 applied to $\Omega \equiv \Sigma:=\partial \mathscr{M}$ with dimension $k:=m-1$ leads to the desired isoperimetric inequality, since it implies $\int_{\partial \mathscr{M}} d S \leq m_{F}^{-(m-1)} \int_{\partial \mathscr{M}} d S_{F}$.

For part (ii) we benefit from our analysis in the proof of part (i), and use [38, Theorem 3.2] to obtain similarly to (3.11) the preliminary Finsler area estimate

$$
\operatorname{area}_{\mathscr{M}}^{F}(X) \leq\left\|\mathcal{A}_{Z}^{F}\right\|_{C^{0}\left(\mathbb{S}^{2}, \mathbb{R}^{3}\right)} \sum_{i=1}^{k}\left[\frac{\mathscr{L}\left(\Gamma_{i}\right)^{2}}{4 \pi}+\frac{1}{2} \mathscr{L}\left(\Gamma_{i}\right) \operatorname{dist}\left(a, \Gamma_{i}\right)\right],
$$

where $\mathscr{L}(\Gamma)=\int|\dot{\Gamma}|$ denotes the Euclidean length of curve $\Gamma \subset \mathbb{R}^{3}$, and $\operatorname{dist}(a, \Gamma)$ is the Eulcidean distance of a point $a \in \mathbb{R}^{3}$ to the curve $\Gamma \subset \mathbb{R}^{3}$. To obtain a right-hand side that is completely expressed in terms of Finslerian quantities we use, on the one hand, the estimate (3.14) on the gradient of the Cartan area integrand, and, on the other hand, the following simple argument comparing Euclidean length and distance to the Finslerian ones: by virtue of (3.2) we have

$$
\mathscr{L}(\Gamma)=\int|\dot{\Gamma}| \stackrel{(3.2)}{\leq} \frac{1}{m_{F}} \int F(\dot{\Gamma})=\frac{1}{m_{F}} \mathscr{L}^{F}(\Gamma)
$$

for any curve $\Gamma \in \mathbb{R}^{3}$. Since $\operatorname{dist}(a, \Gamma)$ and $\operatorname{dist}_{F}(a, \Gamma)$ between a point $a \in \mathbb{R}^{3}$ and a curve $\Gamma \subset \mathbb{R}^{3}$ may be expressed by minimizing the respective length functional over all curves connecting $a$ with some point on $\Gamma$ the estimate (3.17) immediately implies also

$$
\operatorname{dist}(a, \Gamma) \leq \frac{1}{m_{F}} \operatorname{dist}_{F}(a, \Gamma) .
$$

Inserting (3.14), (3.17), and (3.18) into (3.16) we obtain the desired isoperimetric inequality (1.8) as stated.

Notice for part (iii) that $F=F(x, y)$ is not a Minkowski metric, since it depends explicitly on $x \in \mathbb{R}^{3}$. The only isoperimetric inequalities for Cartan functionals depending on the position vector as well we are aware of are those of Clarenz and the second author in [11]. 
By Corollary 2.14 we know that the Cartan area integrand $\mathcal{A}^{F}(x, Z)$ generated by the Finsler metric $F=F(x, y)$ is (parametric) elliptic in the sense of Definition 2.12, in particular we have

$$
\lambda\left|\pi_{Z^{\perp}}(\xi)\right|^{2} \leq|Z| \xi \cdot \mathcal{A}_{Z Z}^{F}(x, Z) \xi \leq \Lambda\left|\pi_{Z^{\perp}}(\xi)\right|^{2} \quad \text { for all } Z \in \mathbb{R}^{3} \backslash\{0\}, x \in \overline{B_{1}(0)}, \xi \in \mathbb{R}^{3}
$$

for some constants $0<\lambda \leq \Lambda<\infty$, which is just (2.23) specified to dimension $m=2$ and for $R_{0}=1$. (The upper bound in (3.19) just follows from smoothness of $\mathcal{A}^{F}$ on $\mathbb{R}^{3} \times\left(\mathbb{R}^{3} \backslash\{0\}\right)$ ). Now, (3.19) is exactly condition (E) in [11, p. 618], and since the positive 1-homogeneity holds for $\mathcal{A}^{F}$ in any case, we can apply [11, Theorem 2] to find for the Euclidean area $\operatorname{area}_{B}(X)$ of $X(B)$

$$
\operatorname{area}_{B}(X) \leq R \frac{2 C\left(\mathcal{A}^{F}\right) \frac{\Lambda}{\lambda}\left[\int_{\Gamma} \kappa d s-2 \pi\right]+\sqrt{\frac{\Lambda}{\lambda}} \mathscr{L}(\Gamma)}{2-R h_{F}},
$$

if

$$
h_{F}:=C\left(\mathcal{A}^{F}\right)\left(1+\left\|\mathcal{A}_{x Z}^{F}\right\|_{\infty}^{2}\right)+\frac{1}{\lambda}\left\|\mathcal{A}_{x Z}^{F}\right\|_{\infty}<2,
$$

where $\left\|\mathcal{A}_{x Z}^{F}\right\|_{\infty}:=\left\|\sum_{i=1}^{3} \mathcal{A}_{x_{i} Z_{i}}^{F}(\cdot, \cdot)\right\|_{C^{0}\left(\overline{B_{1}(0)} \times \mathbb{S}^{2}\right)}$. Here,

$$
C\left(\mathcal{A}^{F}\right):=C_{x}\left(\mathcal{A}^{F}\right)+C_{Z}\left(\mathcal{A}^{F}\right)\left(1+\frac{\Lambda}{2 \lambda^{3}}\right)
$$

is a constant introduced in [11, p. 628], where $C_{x}\left(\mathcal{A}^{F}\right)$ and $C_{Z}\left(\mathcal{A}^{F}\right)$ are bounds on the $x$-derivative, and the $Z$-derivative of the expression

$$
l^{\mathcal{A}^{F}}(x, Z):=\frac{\mathcal{A}_{Z Z}^{F}(x, Z)}{\sqrt{\left.\operatorname{det} \mathcal{A}_{Z Z}^{F}(x, Z)\right|_{Z^{\perp}}}}+\frac{Z}{|Z|} \otimes \frac{Z}{|Z|},
$$

(cf. formulae (35),(38) that lead to Proposition 2.4 (ii) in [11]). We need to investigate all quantities that enter the definition (3.21) of $h_{F}$, since we need to verify its smallness.

Since the denominator in (3.22) is bounded by $\lambda$ from below by virtue of (3.19) we find that for each fixed $x \in \overline{B_{1}(0)} \subset \mathbb{R}^{3}$

$$
\varrho_{1}\left(l^{\mathcal{A}^{F}}(x, \cdot)-l^{\mathcal{A}^{E}}(\cdot)\right) \longrightarrow 0 \quad \text { as } \varrho_{3}\left(\mathcal{A}^{F}(x, \cdot)-\mathcal{A}^{E}(\cdot)\right) \rightarrow 0,
$$

where we refer to the definition of the spherical seminorms in (2.15) and to our notation of the Euclidean metric $E(y)=|y|$ on $\mathbb{R}^{3}$. Analogously to the proof of part (ii) of Theorem 1.1 we can show that for given $\epsilon>0$ we find $\delta=\delta(\epsilon)$ such that the inequality (1.10) implies that

$$
\varrho_{1}\left(l^{\mathcal{A}^{F}}(x, \cdot)-l^{\mathcal{A}^{E}}(\cdot)\right)<\epsilon \quad \text { for all } x \in \overline{B_{1}(0)} .
$$

One easily checks in (3.22) that $l^{\mathcal{A}^{E}}(Z)=\operatorname{Id}_{\mathbb{R}^{3}}$ so that $l_{Z}^{\mathcal{A}^{E}}=0$, and we obviously have no $x$ dependence in $l^{\mathcal{A}^{E}}$, hence $l_{x}^{\mathcal{A}^{E}}=0$. In addition, since $\mathcal{A}^{E}$ does not explicitly depend on $x$ we have $\mathcal{A}_{x Z}^{E}=0$. Consequently, by (3.23), we can choose $\delta_{1}$ sufficiently small such that for all $\delta \in\left(0, \delta_{1}\right)$ for which (1.10) holds, we have

$$
\left\|\mathcal{A}_{x Z}^{F}\right\|_{\infty}^{2}<\min \{1, \lambda\} \quad \text { and } \quad C\left(\mathcal{A}^{F}\right)\left(1+\left\|\mathcal{A}_{x Z}^{F}\right\|_{\infty}^{2}\right)<1,
$$

so that (3.21) is satisfied. Therefore, we have indeed the preliminary isoperimetric inequality (3.20). Since we assumed $R \leq 1$, we can omit $R$ in the denominator. The simple estimates

$$
\operatorname{area}_{B}^{F}(X) \leq M_{F}^{* 2} \operatorname{area}_{B}(X) \quad \text { and } \quad \mathscr{L}(\Gamma) \leq \mathscr{L}^{F}(\Gamma) / m_{F}^{*}
$$


(cf. Lemma 2.1 and (3.17)) lead to the desired isoperimetric inequality (1.9), where we have set

$$
c_{1}(F):=\frac{2 C\left(\mathcal{A}^{F}\right) \frac{\Lambda}{\lambda}}{2-h_{F}} \quad \text { and } \quad c_{2}(F):=\frac{\sqrt{\Lambda / \lambda}}{2-h_{F}},
$$

which also explains why $c_{1}$ vanishes and $c_{2}=1 / 2$ if $F=E(y)=|y|$ since $C\left(\mathcal{A}^{E}\right)=0$ and $h_{F}=h_{E}=0$ verifying our remarks following Theorem 1.5.

Existence and uniqueness of Finsler-minimal immersions spanning given boundary contours. Up to now there are only very few results guaranteeing the existence of immersed surfaces minimizing a general Cartan functional and spanning a given boundary contour. We will draw from White's existence result under the condition of extreme boundary curves [37] to prove with our results of Section 2 the existence of embedded Finsler-minimal disks spanning such a given boundary curve in general Finsler spaces with metric $F=F(x, y)$. In the Minkowski case, $F=F(y)$, we will benefit from a simpler existence and uniqueness result of Winklmann [39].

Let us first explain the notion of Finsler area mean convexity in our assumption in part (i) of Theorem 1.6 adopting the variational characterization that White [37] used for Cartan functionals but without using Finsler-mean curvature in our context to avoid the ambivalent choice of a suitable Finsler normal; see also [4] for an equivalent notion for Cartan integrands that do not depend on position.

Definition 3.1 (Inward-variations and Finsler area mean convexity). Let $Y: \partial \Sigma \rightarrow \mathbb{R}^{3}$ be the injection of the boundary $\partial \Sigma$ of a smooth 3-dimensional submanifold $\Sigma \subset \mathbb{R}^{3}$ into $\mathbb{R}^{3}$.

(i) A smooth mapping $\tilde{Y}:(-\varepsilon, \varepsilon) \times \partial \Sigma \rightarrow \mathbb{R}^{3}$ for some $\varepsilon>0$ is called an inward variation of $\partial \Sigma$ if $\tilde{Y}(0, \cdot)=Y(\cdot)$ on $\partial \Sigma$, with a smooth variation vector field $V(\cdot):=\left.\frac{d}{d t}\right|_{t=0} \tilde{Y}(t, \cdot): \partial \Sigma \rightarrow \mathbb{R}^{3}$, not identically zero, that satisfies for every $u \in \partial \Sigma$ either $V(u)=0$ or $V(u) \notin T_{u} \partial \Sigma$ and $V(u)=c^{\prime}(s)$ for some smooth curve $c:[0, \delta) \rightarrow \Sigma, \delta>0$, with $c(0)=u$.

(ii) If $\mathbb{R}^{3}$ is equipped with a Finsler metric $F=F(x, y)$ then $\Sigma \subset \mathbb{R}^{3}$ is said to be strictly Finsler area mean convex if

$$
\left.\frac{d}{d t}\right|_{t=0} \operatorname{area}_{\partial \Sigma}^{F}(\tilde{Y}(t, \cdot))<0
$$

for all inward variations of $\partial \Sigma$.

Proof of TheOREM 1.6. The explicit representation of Finsler area (2.5) in Theorem $2.2 \mathrm{im}-$ plies that a strictly Finsler area mean convex 3-dimensional submanifold $\Sigma \subset \mathbb{R}^{3}$ with boundary $\partial \Sigma$ is strictly $\mathcal{A}^{F}$-convex in the sense of White [37, items $\left.1.3 \& 1.5\right]$. (Notice that our smoothness assumption on $\Sigma$ implies the strict pointwise inequality required for the normal component of White's $\mathcal{A}^{F}$-mean curvature $[37,1.5]$ even everywhere, not only almost everywhere. White's more general class of Lipschitz variations (see [37, 1.2]), on the other hand, lead to the same variational equations for Cartan functionals as, e.g., in the work of Clarenz et al. [8-10], and therefore to Finsler minimal surfaces in our context.) White's ellipticity assumption [37, pp. 413,414] on the Cartan integrand $\mathcal{A}^{F}$ reads as the uniform convexity of the $\mathcal{A}^{F}(x, \cdot)$-unit ball $\left\{Z \in \mathbb{R}^{3}: \mathcal{A}^{F}(x, Z) \leq 1\right\}$ for each $x$ in $\mathbb{R}^{3}$, which according to [18, p. 72] is satisfied if $\mathcal{A}^{F}$ is parametric elliptic in the sense of Definition 2.12. That this is indeed the case is stated in Corollary 2.14. Hence White's existence result [37, version of Theorem 3.4 on p. 425] is applicable in our context, which in particular leads to the first statement in part (i) of Theorem 1.6. 
The second statement in part (i) is stated in [37, version of Theorem 3.4 on p. 413] and follows from the fact that for a Minkowski metric $F=F(y)$ on $\mathbb{R}^{3}$ smooth convex bodies $\Sigma \subset \mathbb{R}^{3}$ are Finsler area mean convex; see also the other examples of Bergner and Fröhlich in [4, p. 368] whose concept of weighted mean convexity coincides with the one of White in case of Cartan integrands without $x$-dependence.

For part (ii) we refer to [39, Corollary 1.3], where we have to restrict to a Minkowski metric $F=F(y)$ since then the corresponding Cartan area functional $\mathcal{A}^{F}$ depends on $Z$ only, so that the Finsler area

$$
\operatorname{area}_{\Omega}^{F}(X)=\int_{\Omega} \mathcal{A}^{F}\left(X_{u^{1}} \wedge X_{u^{2}}\right) d u^{1} \wedge d u^{2}, \quad \Omega \subset \mathbb{R}^{2},
$$

belongs to the Cartan functionals considered in [39]. The relevant homogeneity and ellipticity conditions formulated in [39, p. 269] are satisfied by $\mathcal{A}^{F}$ as we have observed before; see, e.g., (3.4), and Finsler-minimal immersions are exactly $\mathcal{A}^{F}$-minimal immersions in the language of Clarenz et al. and Winklmann, which means that it has vanishing $\mathcal{A}^{F}$-mean curvature $H_{\mathcal{A}^{F}}=0$; see [39, p. 270].

\section{References}

[1] J. C. Álvarez Paiva and G. Berck. What is wrong with the Hausdorff measure in Finsler spaces. Adv. Math., 204(2):647-663, 2006.

[2] T. N. Bailey, M. G. Eastwood, A. R. Gover, and L. J. Mason. Complex analysis and the Funk transform. J. Korean Math. Soc., 40(4):577-593, 2003. Sixth International Conference on Several Complex Variables (Gyeongju, 2002).

[3] D. Bao, S.-S. Chern, and Z. Shen. An introduction to Riemann-Finsler geometry, volume 200 of Graduate Texts in Mathematics. Springer-Verlag, New York, 2000.

[4] Matthias Bergner and Steffen Fröhlich. Existence, uniqueness and graph representation of weighted minimal hypersurfaces. Ann. Global Anal. Geom., 36(4):363-373, 2009.

[5] Herbert Busemann. Intrinsic area. Ann. of Math. (2), 48:234-267, 1947.

[6] Herbert Busemann. A theorem on convex bodies of the Brunn-Minkowski type. Proc. Nat. Acad. Sci. U. S. A., 35:27-31, 1949.

[7] Shiing-Shen Chern and Zhongmin Shen. Riemann-Finsler geometry, volume 6 of Nankai Tracts in Mathematics. World Scientific Publishing Co. Pte. Ltd., Hackensack, NJ, 2005.

[8] Ulrich Clarenz. Sätze über Extremalen zu parametrischen Funktionalen. PhD thesis, Univ. of Bonn, 1999.

[9] Ulrich Clarenz. Enclosure theorems for extremals of elliptic parametric functionals. Calc. Var. Partial Differential Equations, 15(3):313-324, 2002.

[10] Ulrich Clarenz and Heiko von der Mosel. Compactness theorems and an isoperimetric inequality for critical points of elliptic parametric functionals. Calc. Var. Partial Differential Equations, 12(1):85-107, 2001.

[11] Ulrich Clarenz and Heiko von der Mosel. Isoperimetric inequalities for parametric variational problems. Ann. Inst. H. Poincaré Anal. Non Linéaire, 19(5):617-629, 2002. 
[12] Ningwei Cui and Yi-Bing Shen. Bernstein type theorems for minimal surfaces in $(\alpha, \beta)$-space. Publ. Math. Debrecen, 74(3-4):383-400, 2009.

[13] Ningwei Cui and Yi-Bing Shen. Minimal rotational hypersurfaces in Minkowski $(\alpha, \beta)$-space. Geom. Dedicata, 151:27-39, 2011.

[14] Ulrich Dierkes, Stefan Hildebrandt, Albrecht Küster, and Ortwin Wohlrab. Minimal surfaces. I, volume 295 of Grundlehren der Mathematischen Wissenschaften [Fundamental Principles of Mathematical Sciences]. Springer-Verlag, Berlin, 1992. Boundary value problems.

[15] Lawrence C. Evans and Ronald F. Gariepy. Measure theory and fine properties of functions. Studies in Advanced Mathematics. CRC Press, Boca Raton, FL, 1992.

[16] Paul Funk. Über eine geometrische Anwendung der Abelschen Integralgleichung. Math. Ann., 77(1):129-135, 1915.

[17] Richard J. Gardner. Geometric tomography, volume 58 of Encyclopedia of Mathematics and its Applications. Cambridge University Press, Cambridge, 1995.

[18] Mariano Giaquinta and Stefan Hildebrandt. Calculus of variations. II, volume 311 of Grundlehren der Mathematischen Wissenschaften [Fundamental Principles of Mathematical Sciences]. Springer-Verlag, Berlin, 1996. The Hamiltonian formalism.

[19] David Gilbarg and Neil S. Trudinger. Elliptic partial differential equations of second order. Classics in Mathematics. Springer-Verlag, Berlin, 2001. Reprint of the 1998 edition.

[20] H. Groemer. Geometric applications of Fourier series and spherical harmonics, volume 61 of Encyclopedia of Mathematics and its Applications. Cambridge University Press, Cambridge, 1996.

[21] Sigurdur Helgason. The Radon transform, volume 5 of Progress in Mathematics. Birkhäuser Boston, Mass., 1980.

[22] Sigurdur Helgason. Groups and geometric analysis, volume 113 of Pure and Applied Mathematics. Academic Press Inc., Orlando, FL, 1984. Integral geometry, invariant differential operators, and spherical functions.

[23] Stefan Hildebrandt and Friedrich Sauvigny. An energy estimate for the difference of solutions for the $n$-dimensional equation with prescribed mean curvature and removable singularities. Analysis (Munich), 29(2):141-154, 2009.

[24] Stefan Hildebrandt and Heiko von der Mosel. Dominance functions for parametric Lagrangians. In Geometric analysis and nonlinear partial differential equations, pages 297-326. Springer, Berlin, 2003.

[25] Stefan Hildebrandt and Heiko von der Mosel. Conformal representation of surfaces, and Plateau's problem for Cartan functionals. Riv. Mat. Univ. Parma (7), 4*:1-43, 2005.

[26] H. B. Jenkins. On two-dimensional variational problems in parametric form. Arch. Rational. Mech. Anal., 8:181-206, 1961.

[27] Patrick Overath. Minimal immersions in Finsler spaces. PhD thesis, RWTH Aachen University, Feb. 2014. 
[28] Patrick Overath and Heiko von der Mosel. Plateau's problem in Finsler 3-space. Manuscripta Mathematica, 143:273-316, 2014.

[29] Johann Radon. Über die Bestimmung von Funktionen durch ihre Integralwerte längs gewisser Mannigfaltigkeiten. In 75 years of Radon transform (Vienna, 1992), Conf. Proc. Lecture Notes Math. Phys., IV, pages 324-339. Int. Press, Cambridge, MA, 1994.

[30] Klaus Räwer. Stabile Extremalen parametrischer Doppelintegrale in $\mathbb{R}^{3}$. PhD thesis, Univ. of Bonn, 1993.

[31] Walter Rudin. Functional analysis. McGraw-Hill Book Co., New York, 1973. McGraw-Hill Series in Higher Mathematics.

[32] Zhongmin Shen. On Finsler geometry of submanifolds. Math. Ann., 311(3):549-576, 1998.

[33] Leon Simon. On a theorem of de Giorgi and Stampacchia. Math. Z., 155(2):199-204, 1977.

[34] Leon Simon. On some extensions of Bernstein's theorem. Math. Z., 154(3):265-273, 1977.

[35] Marcelo Souza, Joel Spruck, and Keti Tenenblat. A Bernstein type theorem on a Randers space. Math. Ann., 329(2):291-305, 2004.

[36] Marcelo Souza and Keti Tenenblat. Minimal surfaces of rotation in Finsler space with a Randers metric. Math. Ann., 325(4):625-642, 2003.

[37] Brian White. Existence of smooth embedded surfaces of prescribed genus that minimize parametric even elliptic functionals on 3-manifolds. J. Differential Geom., 33(2):413-443, 1991.

[38] Sven Winklmann. Isoperimetric inequalites involving generalized mean curvature. Analysis (Munich), 22(4):393-403, 2002.

[39] Sven Winklmann. Existence and uniqueness of F-minimal surfaces. Ann. Global Anal. Geom., 24(3):269-277, 2003.

[40] Sven Winklmann. A Bernstein result for entire F-minimal graphs. Analysis (Munich), 27(4):375-386, 2007.

PATRICK OVERATH

Institut für Mathematik

RWTH Aachen University

Templergraben 55

D-52062 Aachen

GERMANY

E-mail: overathe

instmath.rwth-aachen.de
HEIKO VON DER MOSEL Institut für Mathematik

RWTH Aachen University

Templergraben 55

D-52062 Aachen

GERMANY

Email: heikod

instmath.rwth-aachen.de 


\section{Reports des Instituts für Mathematik der RWTH Aachen}

[1] Bemelmans J.: Die Vorlesung "Figur und Rotation der Himmelskörper" von F. Hausdorff, WS 1895/96, Universität Leipzig, S 20, März 2005

[2] Wagner A.: Optimal Shape Problems for Eigenvalues, S 30, März 2005

[3] Hildebrandt S. and von der Mosel H.: Conformal representation of surfaces, and Plateau's problem for Cartan functionals, S 43, Juli 2005

[4] Reiter P.: All curves in a $C^{1}$-neighbourhood of a given embedded curve are isotopic, S 8, Oktober 2005

[5] Maier-Paape S., Mischaikow K. and Wanner T.: Structure of the Attractor of the Cahn-Hilliard Equation, S 68, Oktober 2005

[6] Strzelecki P. and von der Mosel H.: On rectifiable curves with $L^{p}$ bounds on global curvature: Self-avoidance, regularity, and minimizing knots, S 35, Dezember 2005

[7] Bandle C. and Wagner A.: Optimization problems for weighted Sobolev constants, S 23, Dezember 2005

[8] Bandle C. and Wagner A.: Sobolev Constants in Disconnected Domains, S 9, Januar 2006

[9] McKenna P.J. and Reichel W.: A priori bounds for semilinear equations and a new class of critical exponents for Lipschitz domains, S 25, Mai 2006

[10] Bandle C., Below J. v. and Reichel W.: Positivity and anti-maximum principles for elliptic operators with mixed boundary conditions, S 32, Mai 2006

[11] Kyed M.: Travelling Wave Solutions of the Heat Equation in Three Dimensional Cylinders with Non-Linear Dissipation on the Boundary, S 24, Juli 2006

[12] Blatt S. and Reiter P.: Does Finite Knot Energy Lead To Differentiability?, S 30, September 2006

[13] Grunau H.-C., Ould Ahmedou M. and Reichel W.: The Paneitz equation in hyperbolic space, S 22, September 2006

[14] Maier-Paape S., Miller U.,Mischaikow K. and Wanner T.: Rigorous Numerics for the Cahn-Hilliard Equation on the Unit Square, S 67, Oktober 2006

[15] von der Mosel H. and Winklmann S.: On weakly harmonic maps from Finsler to Riemannian manifolds, S 43, November 2006

[16] Hildebrandt S., Maddocks J. H. and von der Mosel H.: Obstacle problems for elastic rods, S 21, Januar 2007

[17] Galdi P. Giovanni: Some Mathematical Properties of the Steady-State Navier-Stokes Problem Past a ThreeDimensional Obstacle, S 86, Mai 2007

[18] Winter N.: $W^{2, p}$ and $W^{1, p}$-estimates at the boundary for solutions of fully nonlinear, uniformly elliptic equations, S 34, Juli 2007

[19] Strzelecki P., Szumańska M. and von der Mosel H.: A geometric curvature double integral of Menger type for space curves, S 20, September 2007

[20] Bandle C. and Wagner A.: Optimization problems for an energy functional with mass constraint revisited, S 20, März 2008

[21] Reiter P., Felix D., von der Mosel H. and Alt W.: Energetics and dynamics of global integrals modeling interaction between stiff filaments, S 38, April 2008

[22] Belloni M. and Wagner A.: The $\infty$ Eigenvalue Problem from a Variational Point of View, S 18, Mai 2008

[23] Galdi P. Giovanni and Kyed M.: Steady Flow of a Navier-Stokes Liquid Past an Elastic Body, S 28, Mai 2008

[24] Hildebrandt S. and von der Mosel H.: Conformal mapping of multiply connected Riemann domains by a variational approach, S 50, Juli 2008

[25] Blatt S.: On the Blow-Up Limit for the Radially Symmetric Willmore Flow, S 23, Juli 2008

[26] Müller F. and Schikorra A.: Boundary regularity via Uhlenbeck-Rivière decomposition, S 20, Juli 2008

[27] Blatt S.: A Lower Bound for the Gromov Distortion of Knotted Submanifolds, S 26, August 2008

[28] Blatt S.: Chord-Arc Constants for Submanifolds of Arbitrary Codimension, S 35, November 2008

[29] Strzelecki P., Szumańska M. and von der Mosel H.: Regularizing and self-avoidance effects of integral Menger curvature, S 33, November 2008

[30] Gerlach H. and von der Mosel H.: Yin-Yang-Kurven lösen ein Packungsproblem, S 4, Dezember 2008

[31] Buttazzo G. and Wagner A.: On some Rescaled Shape Optimization Problems, S 17, März 2009

[32] Gerlach H. and von der Mosel H.: What are the longest ropes on the unit sphere?, S 50, März 2009

[33] Schikorra A.: A Remark on Gauge Transformations and the Moving Frame Method, S 17, Juni 2009

[34] Blatt S.: Note on Continuously Differentiable Isotopies, S 18, August 2009

[35] Knappmann K.: Die zweite Gebietsvariation für die gebeulte Platte, S 29, Oktober 2009

[36] Strzelecki P. and von der Mosel H.: Integral Menger curvature for surfaces, S 64, November 2009

[37] Maier-Paape S., Imkeller P.: Investor Psychology Models, S 30, November 2009

[38] Scholtes S.: Elastic Catenoids, S 23, Dezember 2009

[39] Bemelmans J., Galdi G.P. and Kyed M.: On the Steady Motion of an Elastic Body Moving Freely in a Navier-Stokes Liquid under the Action of a Constant Body Force, S 67, Dezember 2009

[40] Galdi G.P. and Kyed M.: Steady-State Navier-Stokes Flows Past a Rotating Body: Leray Solutions are Physically Reasonable, S 25, Dezember 2009 
[41] Galdi G.P. and Kyed M.: Steady-State Navier-Stokes Flows Around a Rotating Body: Leray Solutions are Physically Reasonable, S 15, Dezember 2009

[42] Bemelmans J., Galdi G.P. and Kyed M.: Fluid Flows Around Floating Bodies, I: The Hydrostatic Case, S 19, Dezember 2009

[43] Schikorra A.: Regularity of n/2-harmonic maps into spheres, S 91, März 2010

[44] Gerlach H. and von der Mosel H.: On sphere-filling ropes, S 15, März 2010

[45] Strzelecki P. and von der Mosel H.: Tangent-point self-avoidance energies for curves, S 23, Juni 2010

[46] Schikorra A.: Regularity of n/2-harmonic maps into spheres (short), S 36, Juni 2010

[47] Schikorra A.: A Note on Regularity for the $n$-dimensional H-System assuming logarithmic higher Integrability, S 30, Dezember 2010

[48] Bemelmans J.: Über die Integration der Parabel, die Entdeckung der Kegelschnitte und die Parabel als literarische Figur, S 14, Januar 2011

[49] Strzelecki P. and von der Mosel H.: Tangent-point repulsive potentials for a class of non-smooth $m$-dimensional sets in $\mathbb{R}^{n}$. Part I: Smoothing and self-avoidance effects, S 47, Februar 2011

[50] Scholtes S.: For which positive $p$ is the integral Menger curvature $\mathcal{M}_{p}$ finite for all simple polygons, S 9, November 2011

[51] Bemelmans J., Galdi G. P. and Kyed M.: Fluid Flows Around Rigid Bodies, I: The Hydrostatic Case, S 32, Dezember 2011

[52] Scholtes S.: Tangency properties of sets with finite geometric curvature energies, S 39, Februar 2012

[53] Scholtes S.: A characterisation of inner product spaces by the maximal circumradius of spheres, S 8, Februar 2012

[54] Kolasiński S., Strzelecki P. and von der Mosel H.: Characterizing $W^{2, p}$ submanifolds by p-integrability of global curvatures, S 44, März 2012

[55] Bemelmans J., Galdi G.P. and Kyed M.: On the Steady Motion of a Coupled System Solid-Liquid, S 95, April 2012

[56] Deipenbrock M.: On the existence of a drag minimizing shape in an incompressible fluid, S 23, Mai 2012

[57] Strzelecki P., Szumańska M. and von der Mosel H.: On some knot energies involving Menger curvature, S 30, September 2012

[58] Overath P. and von der Mosel H.: Plateau's problem in Finsler 3-space, S 42, September 2012

[59] Strzelecki P. and von der Mosel H.: Menger curvature as a knot energy, S 41, Januar 2013

[60] Strzelecki P. and von der Mosel H.: How averaged Menger curvatures control regularity and topology of curves and surfaces, S 13, Februar 2013

[61] Hafizogullari Y., Maier-Paape S. and Platen A.: Empirical Study of the 1-2-3 Trend Indicator, S 25, April 2013

[62] Scholtes S.: On hypersurfaces of positive reach, alternating Steiner formulæ and Hadwiger's Problem, S 22, April 2013

[63] Bemelmans J., Galdi G.P. and Kyed M.: Capillary surfaces and floating bodies, S 16, Mai 2013

[64] Bandle, C. and Wagner A.: Domain derivatives for energy functionals with boundary integrals; optimality and monotonicity., S 13, Mai 2013

[65] Bandle, C. and Wagner A.: Second variation of domain functionals and applications to problems with Robin boundary conditions, S 33, Mai 2013

[66] Maier-Paape, S.: Optimal $f$ and diversification, S 7, Oktober 2013

[67] Maier-Paape, S.: Existence theorems for optimal fractional trading, S 9, Oktober 2013

[68] Scholtes, S.: Discrete Möbius Energy, S 11, November 2013

[69] Bemelmans, J.: Optimale Kurven - über die Anfänge der Variationsrechnung, S 22, Dezember 2013

[70] Scholtes, S.: Discrete Thickness, S 12, Februar 2014

[71] Bandle, C. and Wagner A.: Isoperimetric inequalities for the principal eigenvalue of a membrane and the energy of problems with Robin boundary conditions., S 12, März 2014

[72] Overath P. and von der Mosel H.: On minimal immersions in Finsler space., S 26, April 2014 